\title{
New species and new records of camallanid nematodes (Nematoda, Camallanidae) from marine fishes and sea snakes in New Caledonia
}

\author{
František Moravec $^{1, *}$, and Jean-Lou Justine ${ }^{2}$ \\ ${ }^{1}$ Institute of Parasitology, Biology Centre of the Czech Academy of Sciences, Branišovská 31, 37005 České Budějovice, Czech Republic \\ 2 Institut Systématique Évolution Biodiversité (ISYEB), Muséum National d'Histoire Naturelle, CNRS, Sorbonne Université, EPHE, \\ Université des Antilles, rue Cuvier, CP 51, 75005 Paris, France
}

Received 9 October 2019, Accepted 4 November 2019, Published online 20 November 2019

\begin{abstract}
Recent examinations of camallanid nematodes (Camallanidae) from marine fishes off New Caledonia, collected in the years 2003-2011, revealed the presence of the following five new species of Procamallanus Baylis, 1923, all belonging to the subgenus Spirocamallanus Olsen, 1952: Procamallanus (Spirocamallanus) dispar n. sp. from the common ponyfish Leiognathus equulus (type host) and the striped ponyfish Aurigequula fasciata (both Leiognathidae, Perciformes); Procamallanus (Spirocamallanus) bothi n. sp. from the leopard flounder Bothus pantherinus (Bothidae, Pleuronectiformes); Procamallanus (Spirocamallanus) hexophtalmatis n. sp. from the speckled sandperch Parapercis hexophtalma (Pinguipedidae, Perciformes); Procamallanus (Spirocamallanus) synodi $\mathrm{n}$. sp. from the sand lizardfish Synodus dermatogenys (Synodontidae, Aulopiformes); and Procamallanus (Spirocamallanus) thalassomatis n. sp. from the yellow-brown wrasse Thalassoma lutescens (Labridae, Perciformes). These are described based on light and scanning electron microscopical (SEM) studies. An additional three congeneric nematodes unidentifiable to species are reported from perciform fishes and a shark: Procamallanus (Spirocamallanus) sp. 3 of Moravec et al., 2006, Procamallanus (Spirocamallanus) sp. 1, and Procamallanus (Spirocamallanus) sp. 2. Ten fish species are recorded as new hosts for Camallanus carangis Olsen, 1954. Two camallanids, Procamallanus (Spirocamallanus) sp. 3 (subgravid female) and Camallanus carangis (fourth-stage larva) were also found in the digestive tract of the New Caledonian sea krait Laticauda saintgironsi, serving apparently as postcyclic and paratenic hosts, respectively, for these fish nematodes.
\end{abstract}

Key words: Nematoda, Helminth parasite, Camallanoidea, Aulopiformes, Carcharhiniformes, Perciformes, Pleuronectiformes, Serpentes, South Pacific.

\begin{abstract}
Résumé - Nouvelles espèces et nouveaux signalements de nématodes camallanidés (Nematoda, Camallanidae) provenant de poissons marins et de serpents de mer en Nouvelle-Calédonie. L'étude récente de nématodes camallanidés (Camallanidae) de poissons marins capturés au large de la Nouvelle-Calédonie, recueillis de 2003 à 2011, a révélé la présence des cinq nouvelles espèces suivantes de Procamallanus Baylis, 1923, toutes appartenant au sous-genre Spirocamallanus Olsen, 1952 : Procamallanus (Spirocamallanus) dispar n. sp. de Leiognathus equulus (hôte-type) et de Aurigequula fasciata (tous deux Leiognathidae, Perciformes) ; Procamallanus (Spirocamallanus) bothi n. sp. de Bothus pantherinus (Bothidae, Pleuronectiformes) ; Procamallanus (Spirocamallanus) hexophtalmatis n. sp. de Parapercis hexophtalma (Pinguipedidae, Perciformes) ; Procamallanus (Spirocamallanus) synodi n. sp. de Synodus dermatogenys (Synodontidae, Aulopiformes) ; et Procamallanus (Spirocamallanus) thalassomatis n. sp. de Thalassoma lutescens (Labridae, Perciformes). Ces espèces sont décrites sur la base d'études réalisées au microscope électronique à balayage et au microscope photonique. Trois autres nématodes congénères non identifiables au niveau spécifique sont signalés chez des poissons perciformes et un requin : Procamallanus (Spirocamallanus) sp. 3 de Moravec et al., 2006, Procamallanus (Spirocamallanus) sp. 1 et Procamallanus (Spirocamallanus) sp. 2. Dix espèces de poissons sont rapportées comme nouveaux hôtes pour Camallanus carangis Olsen, 1954. Deux camallanidés, Procamallanus (Spirocamallanus) sp. 3 (femelles subgravides) et Camallanus carangis (larve du quatrième stade) ont également été trouvés dans le tube digestif du serpent marin de Nouvelle-Calédonie, Laticauda saintgironsi, qui semble servir respectivement d'hôte post-cyclique et d'hôte paraténique pour ces nématodes de poissons.
\end{abstract}

\footnotetext{
*Corresponding author: moravec@paru. cas. cz
} 


\section{Introduction}

Nematodes of the family Camallanidae Railliet et Henry, 1915 , characterized by a well-developed, usually orangecoloured buccal capsule and a life cycle involving a copepod intermediate host, are mostly gastrointestinal, blood-sucking parasites of marine, brackish-water and freshwater fishes and, less often, of amphibians and aquatic reptiles (turtles, snakes) [1, 10, 41]. Although camallanids are frequent parasites of Indo-Pacific fishes, where many species have been reported, data on these nematodes in New Caledonian waters are scarce. To date, only the following nominal species of camallanids have been recorded from New Caledonia: Camallanus cotti Fujita, 1927 (an introduced species) and Procamallanus (Procamallanus) pacificus Moravec, Justine, Würtz, Taraschewski et Sasal, 2006 in freshwater fishes and Camallanus carangis Olsen, 1954, Procamallanus (Procamallanus) annulatus Yamaguti, 1955, Procamallanus (Spirocamallanus) longus Moravec, Justine et Rigby, 2006, P. (S.) monotaxis (Olsen, 1952), P. (S.) sinespinis Moravec et Justine, 2017 and $P$. (S.) variolae Moravec, Justine et Rigby, 2006 in marine fishes [12-14, 23-28].

Recent examinations of camallanid nematodes collected by J.-L. Justine and his students in marine fishes from off New Caledonia in the years 2003-2011 revealed the presence of five previously unknown species of Procamallanus Baylis, 1923 (subgenus Spirocamallanus Olsen, 1952) and three forms of the same subgenus unidentifiable to species, and showed several new hosts of one already known species of Camallanus Railliet et Henry, 1915. In addition, two fish camallanids were found in the digestive tract of sea snakes Laticauda saintgironsi Cogger et Heatwole. Results of this study are presented herein.

\section{Materials and methods}

Fish were caught off New Caledonia by various means; those obtained from the fishmarket in Nouméa were very fresh and thus were probably fished in the near vicinity. For fish, we generally used the "wash" method [15]. For sea snakes, as these hosts are emblematic protected species, an indirect sampling method without any effect on the individual survival was used; a gentle massage of the sea krait abdomen provided the stomach content by regurgitation [4]. The regurgitated contents might include recently swallowed fish, which are thus recognizable [43], or, if digestion has already occurred, no recognizable item, as was the case for the samples described in this study. The nematodes for morphological studies were fixed in hot $4 \%$ formalin or $70 \%$ ethanol. For light microscopical examination, they were cleared with glycerine. Drawings were made with the aid of a Zeiss microscope drawing attachment. Specimens used for scanning electron microscopical (SEM) examination were postfixed in $1 \%$ osmium tetroxide (in phosphate buffer), dehydrated through a graded acetone series, criticalpoint-dried and sputter-coated with gold; they were examined using a JEOL JSM-7401F scanning electron microscope at an accelerating voltage of $4 \mathrm{kV}$ (GB low mode). All measurements are in micrometres unless otherwise indicated. The fish nomenclature adopted follows FishBase [7].

\section{Results}

Procamallanus (Spirocamallanus) dispar $n$. sp. Figures 1, 2

urn:lsid:zoobank.org:act:579423E1-64EA-4D20-851164E5A5EB0753

Type host: Common ponyfish Leiognathus equulus (Forsskål) (Leiognathidae, Perciformes).

Other host: Striped ponyfish Aurigequula fasciata (Lacepède) (Leiognathidae, Perciformes).

Site of infection: Intestine.

Type locality: Fishmarket, Nouméa, New Caledonia (collected 1 October 2008).

Prevalence, intensity and details about fish: L. equulus: 3 fish infected/4 fish examined; 2 nematodes per fish (Fish numbers: JNC2644, JNC2645, JNC2647, 1 October 2008). A. fasciata: 1 fish infected/4 fish examined; 1 nematode (Fish number: JNC2923, 29 April 2009). The infected fish were 129-137 $\mathrm{mm}$ in fork length and 62-68 $\mathrm{g}$ in weight (L. equulus), and $132 \mathrm{~mm}$ in length and $63 \mathrm{~g}$ in weight (A. fasciata).

Deposition of type specimens: Muséum National d'Histoire Naturelle, Paris, France (male holotype, female allotype and 1 paratype, MNHN JNC 2644 and JNC2647); Helminthological Collection, Institute of Parasitology, Biology Centre of the Czech Academy of Sciences, České Budějovice, Czech Republic (four male paratypes [three mounted on SEM stub], N-1200).

Etymology: The specific name of this nematode dispar (= different) is the Latin adjective relating to the fact that this species differs from related congeners.

\section{Description}

General: Medium-sized nematodes with finely transversely striated cuticle. Mouth aperture oval, surrounded by 12 submedian cephalic papillae arranged in three circles, each formed by four papillae; papillae of external circle distinctly larger; all papillae accompanied by distinct proximal pore; additional pair of pores situated laterally near edge of oral aperture; pair of small lateral amphids present (Figs. 1D, 2A and 2B). Buccal capsule orange, thick-walled, longer than wide, with simple, well-developed basal ring. Maximum width/length ratio of buccal capsule 1:1.12-1.39. Inner surface of capsule provided with 12-14 spiral ridges in lateral view, 4-6 of them being incomplete (Figs. 1B and 1C). Muscular oesophagus shorter than glandular oesophagus; both parts of oesophagus slightly expanded near their posterior ends (Figs. 1A and 1B). Intestine brown, narrow. Deirids small, simple, with rounded end situated approximately at mid-way between base of buccal capsule and nerve ring (Figs. 1A, 1B, 1E and 2D). Excretory pore located short distance anterior to posterior end of muscular oesophagus (Fig. 1B).

Male (four specimens, including holotype, from L. equulus; measurements of one juvenile specimen from $L$. equulus in parentheses. Measurements of one specimen from A. fasciata in brackets): Length of body 17.95-20.13 (10.47) [16.32] mm, maximum width 258-326 (218) [272]. Buccal capsule including basal ring 81-90 (84) [87] long, its width 66-75 (69) [72]; basal ring 9-12 (9) [9] long and 48-54 (45) [54] 

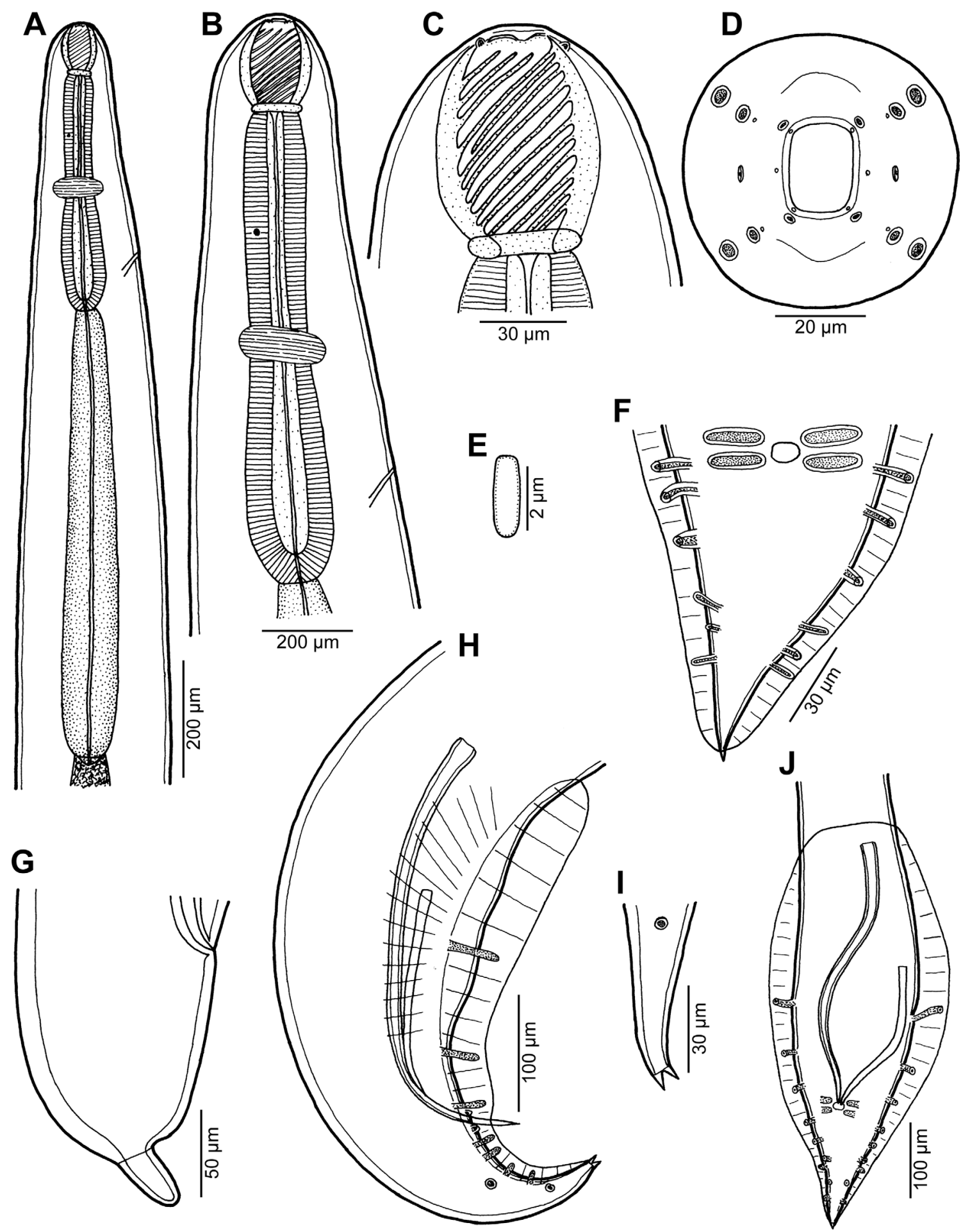

Figure 1. Procamallanus (Spirocamallanus) dispar n. sp. from Leiognathus equulus. (A) Anterior end of male, lateral view; (B) same, enlarged; (C, D) cephalic end of male, lateral and apical views, respectively; (E) deirid; (F) tail of male, ventral view; (G) tail of subgravid female, lateral view; $(\mathrm{H})$ posterior end of male, lateral view; (I) tail tip of juvenile male, lateral view; (J) posterior end of juvenile male, ventral view.

wide. Maximum width/length ratio of buccal capsule 1:1.121.36 (1:1.22) [1:1.21]. Spiral ridges 12-14 (16) [14], 6 (5) [5] of which incomplete. Length of muscular oesophagus 462503 (435) [490], maximum width 69-87 (69) [87]; length of glandular oesophagus 734-884 (503) [653], maximum width 108-126 (84) [126]; length ratio of muscular and glandular oesophagus $1: 1.59-1.85(1: 1.16)$ [1:1.33]. Length of entire oesophagus and buccal capsule representing 6-7 (10) [8]\% of 


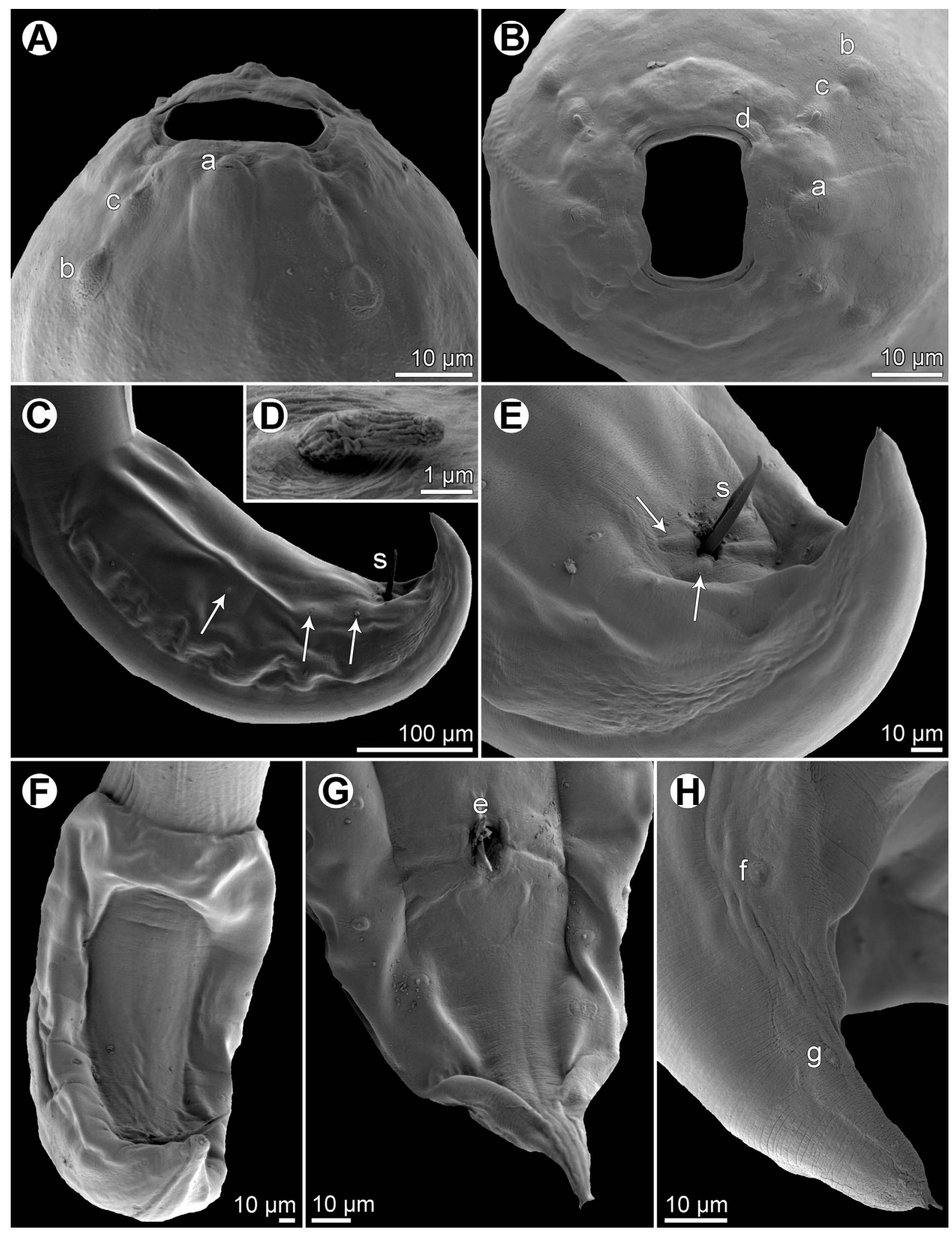

Figure 2. Procamallanus (Spirocamallanus) dispar n. sp., scanning electron micrographs of male. (A, B) Cephalic end, lateral and apical views, respectively; (C) posterior end of juvenile specimen, lateral view (arrows indicate subventral preanal papillae); (D) deirid; (E) tail, ventrolateral view (arrows indicate elongate papillae surrounding cloaca); (F) posterior end, ventral view; (G) tail of juvenile specimen, ventral view; (H) distal part of tail, lateral view. (a) amphid; (b) cephalic papilla of external circle; (c) cephalic papilla of middle circle; (d) cephalic papilla of internal circle; (e) cloacal aperture; (f) lateral postanal papilla; (g) phasmid; (s) spicule. 
body length. Deirids, nerve ring and excretory pore 279-357 (297) [309], 358-326 (272) [299] and 394-462 (490) [-] from anterior extremity, respectively. Posterior end of body ventrally bent, provided with wide, vesiculated caudal alae supported by pedunculate papillae; anteriorly alae interconnected by mound, forming a kind of pseudosucker, and posteriorly reaching to caudal terminal spines (Figs. 1F, 1H-1J, 2C and 2E-2H). Preanal papillae: three pairs of subventral pedunculate papillae, of which second and third pairs closer to each other than first and second pairs; postanal papillae: six pairs of pedunculate papillae, four subventral and two lateral (last pair representing phasmids); additional two pairs of transversely-elongate sessile ventral papillae surrounding cloacal opening (Figs. 1F, 1H, 1J, $2 \mathrm{E}$ and $2 \mathrm{G})$. Spicules unequal, with sharply pointed distal ends; large (right) spicule 426-449 (195) [408] long; small (left) spicule less sclerotized, 180-250 (90) [204] long. Length ratio of spicules 1:1.70-2.42 (1:2.17) [1:2.00]. Gubernaculum absent. Tail conical, 135-195 (138) [135] long, with two (dorsal and ventral) small terminal cuticular spines 3-6 (6) [6] long (Figs. 1F, 1H, 1I, 2G and 2H).

Female (one ovigerous specimen from L. equulus, allotype): Length of body $19.07 \mathrm{~mm}$, maximum width 313 . Buccal capsule including basal ring 96 long and 69 wide; basal ring 9 long and 57 wide. Maximum width/length ratio of buccal capsule 1:1.39. Number of spiral ridges 12 , of which 4 incomplete. Length of muscular oesophagus 462 , maximum width 87 ; length of glandular oesophagus 558, maximum width 114; length ratio of muscular and glandular oesophagus 1:1.21. Length of entire oesophagus and buccal capsule representing $6 \%$ of body length. Deirids, nerve ring and excretory pore 345, 272 and 452, respectively, from anterior extremity. Vulva postequatorial, $9.86 \mathrm{~mm}$ from anterior extremity, at $52 \%$ of body length. Vulval lips not elevated. Uterus filled with numerous eggs. Tail broad, rounded, its posterior end abruptly narrowed to form terminal digit-like protrusion with smooth, rounded tip; length of entire tail 162; digit-like protrusion 36 long, 18 wide (Fig. 1G).

\section{Remarks}

The present specimens from L. equulus and A. fasciata are considered to be conspecific because of their morphological and biometrical similarity and the fact that both of their host species belong to the same fish family. These nematodes belong to the subgenus Spirocamallanus of the genus Procamallanus in the conception of Moravec and Thatcher [32], namely to the group of Spirocamallanus species characterized by the presence of wide caudal alae, three pairs of pedunculate preanal papillae and two unequal spicules, that are mostly parasites of marine fishes [37]. Most species of this group are characterized by the presence of two caudal spikes, one dorsal and one ventral, on a digital projection in the female $[9,46]$, whereas these are lacking only in a few species. According to Petter et al. [38], Rigby and Adamson [39] and Moravec et al. [27], the shape and structure of the female tail appears to be constant within a species of Procamallanus (Spirocamallanus).

By the shape of the female tail and the absence of any terminal spikes, $P$. (S.) dispar n. sp. resembles only $P$. (S.) mexicanus Moravec, Salgado-Maldonado et Caspeta-Mandujano, 2000 from the freshwater cichlid Cichlasoma geddesi (Regan) (Cichlidae) in Mexico [29], P. (S.) murrayensis Johnston et Mawson, 1940 from freshwater perciform fishes Pseudaphritis urvillii (Valenciennes) (Pseudophritidae), Macquaria colonorum (Günther) and M. ambigua (Richardson) (both Percichthyidae) in Australia [11] and $P$. (S.) sinespinis from the marine fish Pomadasys argenteus (Forsskål) (Haemulidae) off New Caledonia [26]. Vicente and Santos [45] did not report the presence of two terminal caudal spines in females of the inadequately described $P$. (S.) macaensis Vicente et Santos, 1972, a parasite of several species of marine fishes in Brazil [16], but these are present according to the later redescription of this species [42].

However, in contrast to the new species, the right spicule of P. murrayensis is distinctly shorter $(290 \mu \mathrm{m} v s 408-449 \mu \mathrm{m})$. The right spicules of $P$. mexicanus and $P$. sinespinis are only slightly longer (456-480 $\mu \mathrm{m}$ and 465-525 $\mu \mathrm{m}$, respectively $v s 408-449 \mu \mathrm{m})$ than those of $P$. dispar, but the number of spiral ridges in their buccal capsules is 10-12 (vs 12-14); in addition, the male tail tip of these two species bears either a single conical cuticular spike (P. mexicanus) or a knob-like structure ( $P$. sinespinis) ( $v s$ two, dorsal and ventral, terminal spikes are present); the female tail of $P$. mexicanus has a different shape, its anterior portion being narrow and conical (vs broad and posteriorly rounded). Moreover, P. mexicanus and $P$. murrayensis are parasites of freshwater fishes, whereas the hosts of $P$. dispar are marine fishes.

Moravec et al. [27] reported a nematode subgravid female, designated as Procamallanus (Spirocamallanus) sp. 3, from the marine fish Scolopsis bilineata (Bloch) (Nemipteridae) from off New Caledonia. The shape of its tail is similar to that of $P$. dispar and also the number (13) of spiral ridges in the buccal capsule corresponds to this species. However, the location of deirids is different (at the level of the nerve ring $v s$ at the mid-way between the buccal capsule and the nerve ring), so that this specimen probably represents another species.

\section{Procamallanus (Spirocamallanus) bothi n. sp. Figures 3, 4}

urn:lsid:zoobank.org:act:CCA32AF1-B9B7-4A1C-99FF2C5A5A53410B

Type host: Leopard flounder Bothus pantherinus (Rüppell) (Bothidae, Pleuronectiformes).

Site of infection: Intestine.

Type locality: Fishmarket, Nouméa, New Caledonia (collected 11 February 2011).

Prevalence, intensity and details about fish: 1 fish infected/ 1 fish examined; 12 nematodes. The infected fish (Fish number: JNC3310) was $234 \mathrm{~mm}$ in fork length and $128 \mathrm{~g}$ in weight.

Deposition of type specimens: Muséum National d'Histoire Naturelle, Paris, France (male holotype, female allotype and eight paratypes, MNHN JNC 3310); Helminthological Collection, Institute of Parasitology, Biology Centre of the Czech Academy of Sciences, České Budějovice, Czech Republic (two paratypes mounted on SEM stub, N-1201).

Etymology: The specific name of this nematode relates to the genitive form of the generic name of the host. 

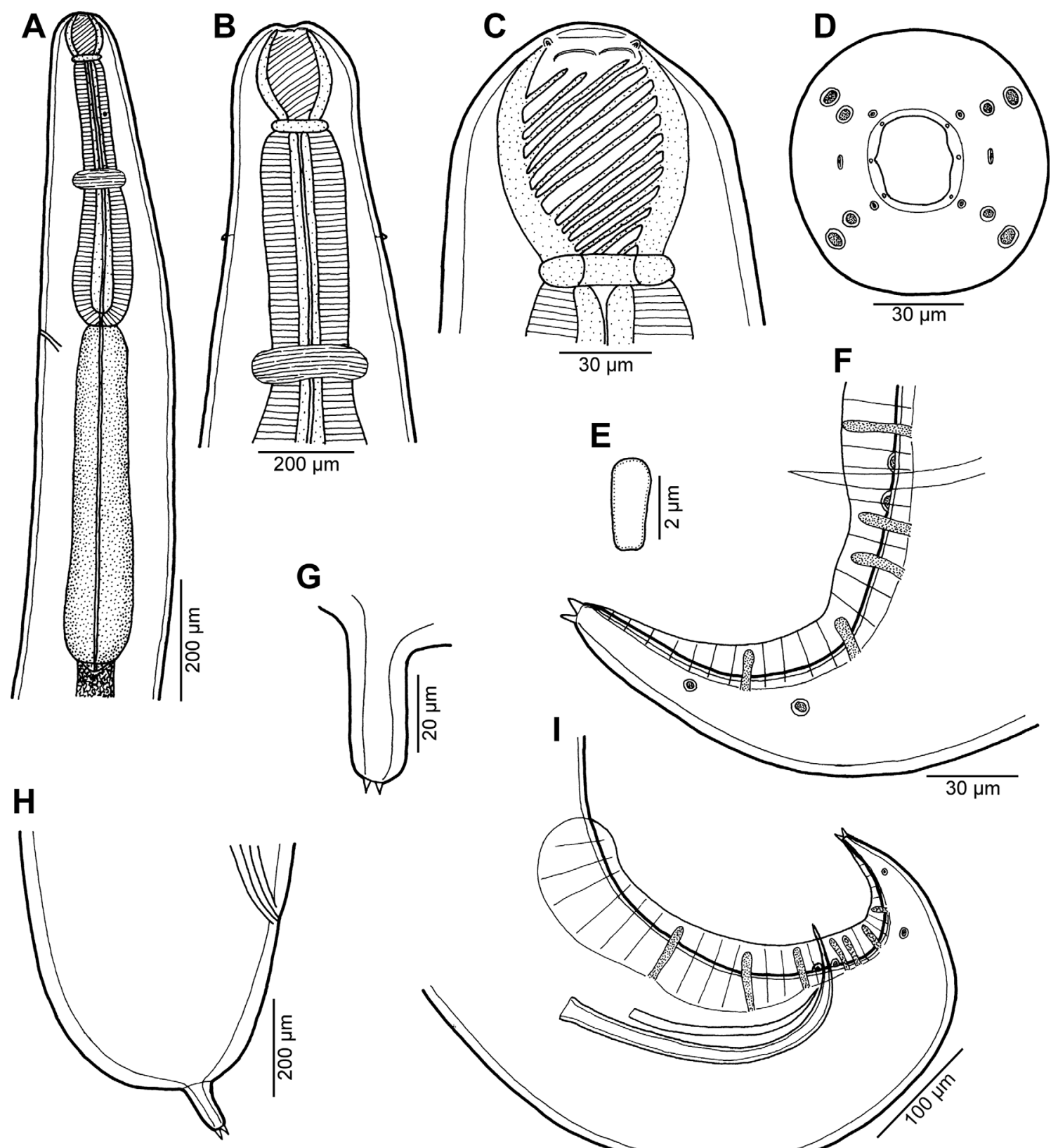

$\mathbf{F}$

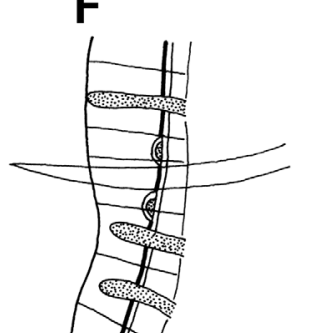




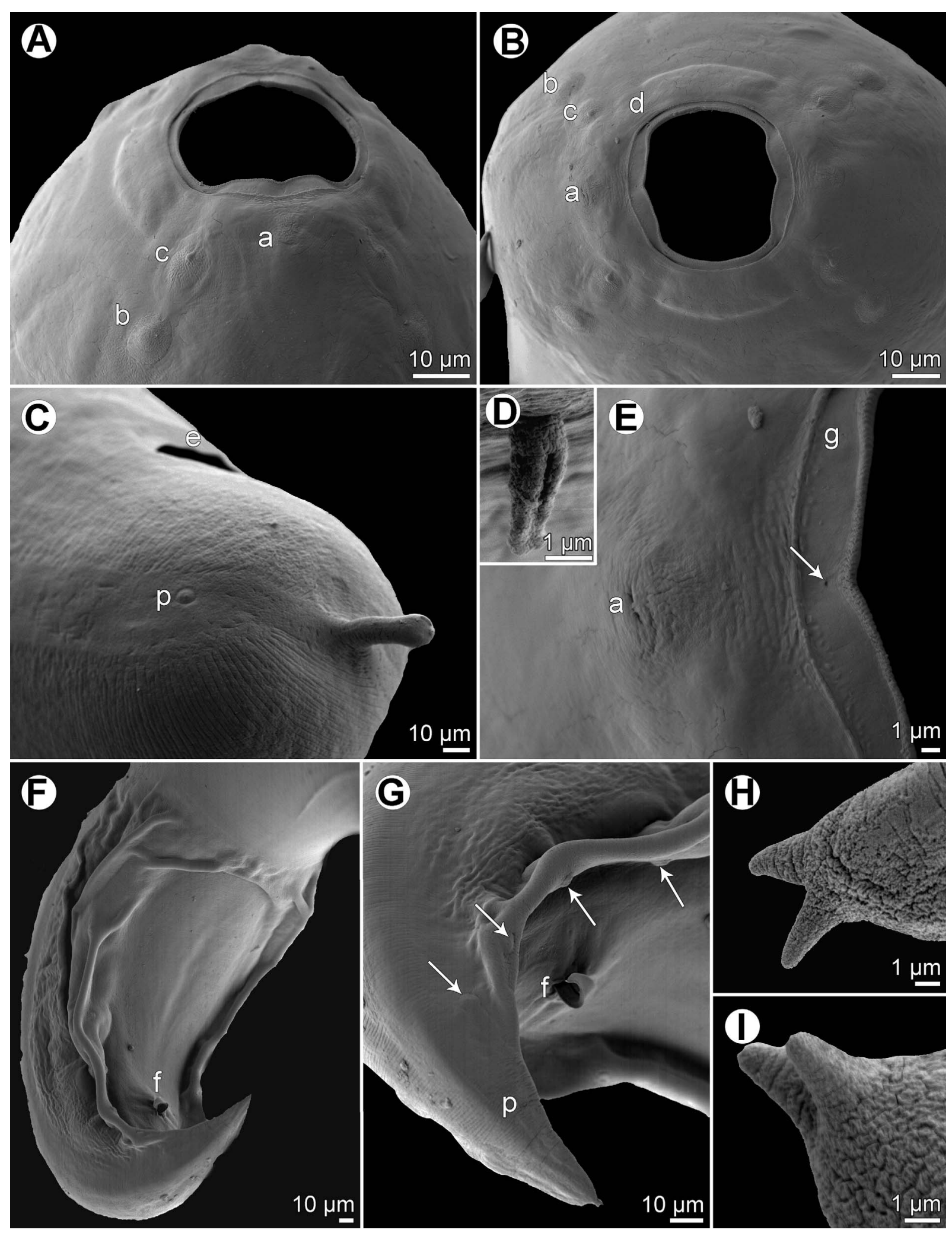

Figure 4. Procamallanus (Spirocamallanus) bothi n. sp., scanning electron micrographs. (A, B) Cephalic end, lateral and apical views, respectively; (C) female tail, sublateral view; (D) deirid; (E) region of amphid, apical view (arrow indicates lateral pore on margin of oral aperture); (F) posterior end of male, subventral view; (G) tail of male, ventrolateral view (arrows indicate pedunculated caudal papillae); (H) tail tip of male (lateral view); (I) tail tip of female, sublateral view). (a) amphid; (b) cephalic papilla of external circle; (c) cephalic papilla of middle circle; (d) cephalic papilla of internal circle; (e) anus; (f) cloacal aperture; (g) margin of oral aperture; (p) phasmid. 
Spiral ridges 14 (14), 6 (5) of which incomplete. Length of muscular oesophagus 476 (490), maximum width 102 (111); length of glandular oesophagus 612 (748), maximum width 123 (156); length ratio of muscular and glandular oesophagus 1:1.29 (1:1.53). Length of entire oesophagus and buccal capsule representing 6 (7)\% of body length. Deirids, nerve ring and excretory pore 201 (195), 313 (313) and 639 (680) from anterior extremity, respectively. Posterior end of body ventrally bent, provided with wide, vesiculated caudal alae supported by pedunculate papillae; anteriorly alae interconnected by mound, forming a kind of pseudosucker, and posteriorly reaching to caudal terminal spines (Figs. 3F, 3I, 4F and 4G). Preanal papillae: three pairs of subventral pedunculate papillae, of which second and third pairs closer to each other than first and second pairs; postanal papillae: six pairs of pedunculate papillae, four subventral and two lateral (last pair representing phasmids); additional two pairs of small, transversely-elongate sessile ventral papillae surrounding cloacal opening (Figs. 3F, 3I and 4G). Spicules unequal, with sharply pointed distal ends; large (right) spicule 267 (270) long; small (left) spicule less sclerotized, 180 (177) long (Fig. 3I). Length ratio of spicules 1:1.48 (1:1.53). Gubernaculum absent. Tail conical, 207 (153) long, with two (dorsal and ventral) small terminal cuticular spines 6 (6) long (Figs. 3F, 3I and 4H).

Female (seven larvigerous specimens; measurements of allotype in parentheses. Measurements of additional three ovigerous specimens in brackets): Length of body 25.64-38.80 (37.92) [15.16-22.77] mm, maximum width 490-762 (734) [299408]. Buccal capsule including basal ring 102-114 (102) [96-105] long and 84-105 (90) [84-99] wide; basal ring 9-12 (9) [9] long and 60-66 (60) [57-69] wide. Maximum width/ length ratio of buccal capsule 1:1.06-1.27 (1:1.13) [1:1.031.25]. Number of spiral ridges 12-18 (18) [12-19], of which 3-5 (5) [3-8] incomplete. Length of muscular oesophagus 571-639 (598) [530-571], maximum width 129-136 (136) [69-111]; length of glandular oesophagus 721-911 (911) [612-748], maximum width 135-177 (177) [102-135]; length ratio of muscular and glandular oesophagus 1:1.22-1.52 (1:1.52) [1:1.15-1.38]. Length of entire oesophagus and buccal capsule representing 4-6 (4) [7-9]\% of body length. Deirids, nerve ring and excretory pore 231-258 (233) [218-231], 340-381 (381) [313-354] and 707-884 (707) [639-680], respectively, from anterior extremity. Vulva mostly pre-equatorial (exceptionally equatorial), 11.37-18.90 (18.90) [7.71-10.36] $\mathrm{mm}$ from anterior extremity, at 36-50 (50) [46-50]\% of body length. Vulval lips not elevated. Vagina directed posteriorly from vulva. Uterus filled with numerous larvae 390-404 long and 21-24 in maximum width, with slender tail [with small amount of eggs]. Tail broad, rounded, its posterior end abruptly narrowed to form digit-like protrusion provided with 2 (2) [2], dorsal and ventral, small terminal cuticular spikes; length of entire tail 190-218 (204) [138-204]; digit-like protrusion 36-51 (39) [21-42] long, 15-18) [12-15] wide, length of spines 3-5 (5) [5] (Figs. 3G, 3H, 4C and 4I).

\section{Remarks}

Nematodes of the present material belong to the morphological group of Procamallanus (Spirocamallanus) species characterized by the presence of wide caudal alae, three pairs of pedunculate preanal papillae, two unequal spicules and two caudal spikes on a digital projection in the female. According to Yooyen et al. [46], in the Indo-Pacific region this group contains 23 nominal species reported mostly from marine fishes. However, the great majority of them are poorly described and should be considered species inquirendae [see also 31,40$]$.

The following nine species of this morphological group from the Indo-Pacific region can be considered valid: $P$. (S.) anguillae Moravec, Taraschewski, Thairungroj Anantaphruti, Maipanich et Laoprasert, 2006 from Anguilla bicolor McClelland (Anguillidae) in Thailand and India [30, 31], P. (S.) gobiomori Moravec, Salgado-Maldonado et Caspeta-Mandujano, 2000 from freshwater Eleotridae in western Mexico [29], P. (S.) guttatusi (Andrade-Salas, PinedaLópez et García-Magaña, 1994) from Siganus guttatus (Bloch) (Siganidae) off the Philippines [17, 22], P. (S.) istiblenni (Noble, 1966) from Istiblennius zebra (Vailland et Sauvage) (Blenniidae) off Hawaii [33, 40], P. (S.) monotaxis (Olsen, 1952) from Lethrinidae off Hawaii, New Caledonia and French Polynesia [25, 35, 39], P. (S.) pereirai (Annereaux, 1946) from Atherinopsis californiensis Girard (Atherinidae) off California, USA [3], P. (S.) rigbyi Yooyen, Moravec et Wongsawad, 2011 from Otolithes ruber (Bloch et Schneider) (Sciaenidae) off Thailand [46], P. (S.) similis Yooyen, Moravec et Wongsawad, 2011 from Sillago sihama (Forsskål) (Sillaginidae) off Thailand [46] and P. (S.) variolae Moravec, Justine et Rigby, 2006 from Variola spp. (Serranidae) off New Caledonia [27].

Of these, as compared with the new species, the right spicule is distinctly longer in P. gobiomori $(318-348 \mu \mathrm{m} v \mathrm{~s}$ 267-270 $\mu \mathrm{m})$, P. pereirai $(430 \mu \mathrm{m})$, P. rigbyi $(315-360 \mu \mathrm{m})$, $P$. similis $(435-492 \mu \mathrm{m})$ and $P$. variolae $(327-357 \mu \mathrm{m})$; moreover, the spiral ridges in the buccal capsule are less numerous in P. gobiomori (8-10 vs 13-19), P. similis (10-12) and $P$. variolae (11-12) and all these five species also differ in the family and order of their fish hosts (Perciformes: Eleotridae, Serranidae, Sciaenidae and Sillaginidae or Atheriniformes: Atherinidae vs Pleuronectiformes: Bothidae). The right spicule of $P$. anguillae is somewhat longer (289-384 $\mu \mathrm{m} v \mathrm{~s}$ 267-270 $\mu \mathrm{m}$ ) than that of $P$. bothi n. sp., the spiral ridges are usually less numerous (10-15 vs 13-19) and both species differ in the type of the host (freshwater eel vs marine flatfish). The length of the right spicule in P. istiblenni and P. monotaxis is rather similar to that in the new species $(263-302 \mu \mathrm{m}$ and 279-315 $\mu \mathrm{m}$, respectively $v s 267-270 \mu \mathrm{m})$, but the spiral ridges are mostly less numerous (12-15 and $10-17$, respectively $v s$ 13-19); deirids of $P$. istiblenni are located in $2 / 3$ of the distance between the base of the buccal capsule and the nerve ring ( $v s$ in the mid-length of this distance) and the excretory pore at the level of the posterior end of the muscular oesophagus (vs somewhat posterior to this level), and the female tail of $P$. istiblenni is more conical as compared with that of $P$. bothi $\mathrm{n}$. sp.; the excretory pore of $P$. monotaxis is located short distance anterior to the posterior margin of the muscular oesophagus ( $v s$ somewhat posterior to the anterior end of the glandular oesophagus). Moreover, the hosts of $P$. istiblenni and $P$. monotaxis belong to different fish families and orders (Perciformes: Blenniidae and Lethrinidae, respectively vs Pleuronectiformes: Bothidae). 

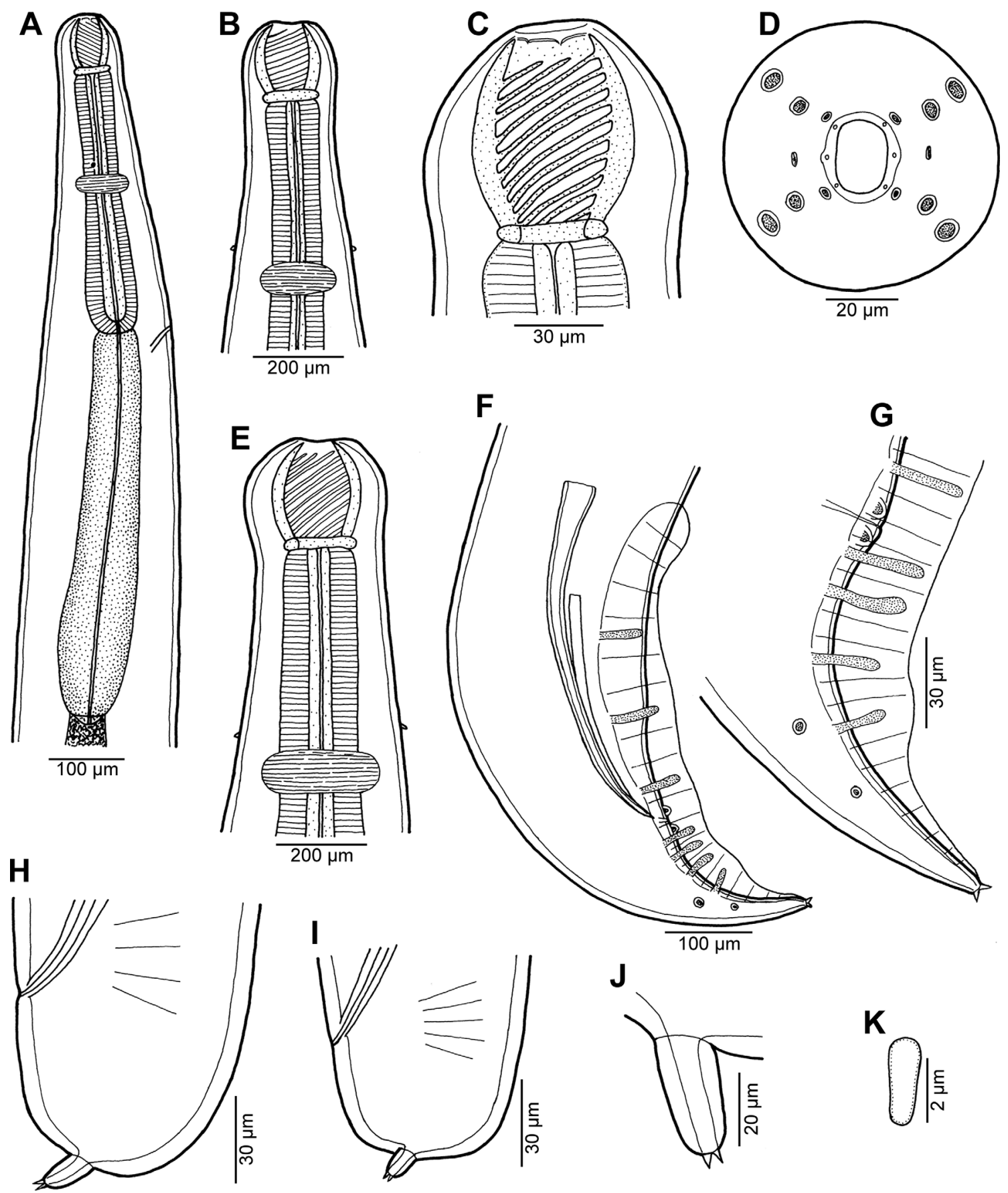

Figure 5. Procamallanus (Spirocamallanus) hexophtalmatis n. sp. from Parapercis hexophtalma. (A, B) Anterior end of male, lateral and dorsoventral views, respectively; (C, D) cephalic end of male, lateral and apical views, respectively; (E) anterior end of gravid female, dorsoventral view; (F) posterior end of male, lateral view; (G) tail of male, lateral view; (H, I) tail of gravid and subgravid female, respectively, lateral views; (J) tail tip of gravid female, lateral view; (K) deirid.

\section{Procamallanus (Spirocamallanus) hexophtalmatis n. sp. Figures 5, 6}

urn:Isid:zoobank.org:act:09400000-B876-4263-82A4A0D2EA576751

Type host: Speckled sandperch Parapercis hexophtalma (Cuvier) (Pinguipedidae, Perciformes).

Site of infection: Intestine.
Type locality: Near Récif Toombo, off Nouméa, New Caledonia, $22^{\circ} 32^{\prime} 934 \mathrm{~S}, 166^{\circ} 28^{\prime} 549 \mathrm{E}$ (collected 4 November 2008).

Prevalence, intensity and details about fish: 1 fish infected/ 2 fish examined; 4 nematodes (Fish number: JNC2736). The infected fish was $185 \mathrm{~mm}$ in fork length and $68 \mathrm{~g}$ in weight.

Deposition of type specimens: Helminthological Collection, Institute of Parasitology, Biology Centre of the Czech Academy 


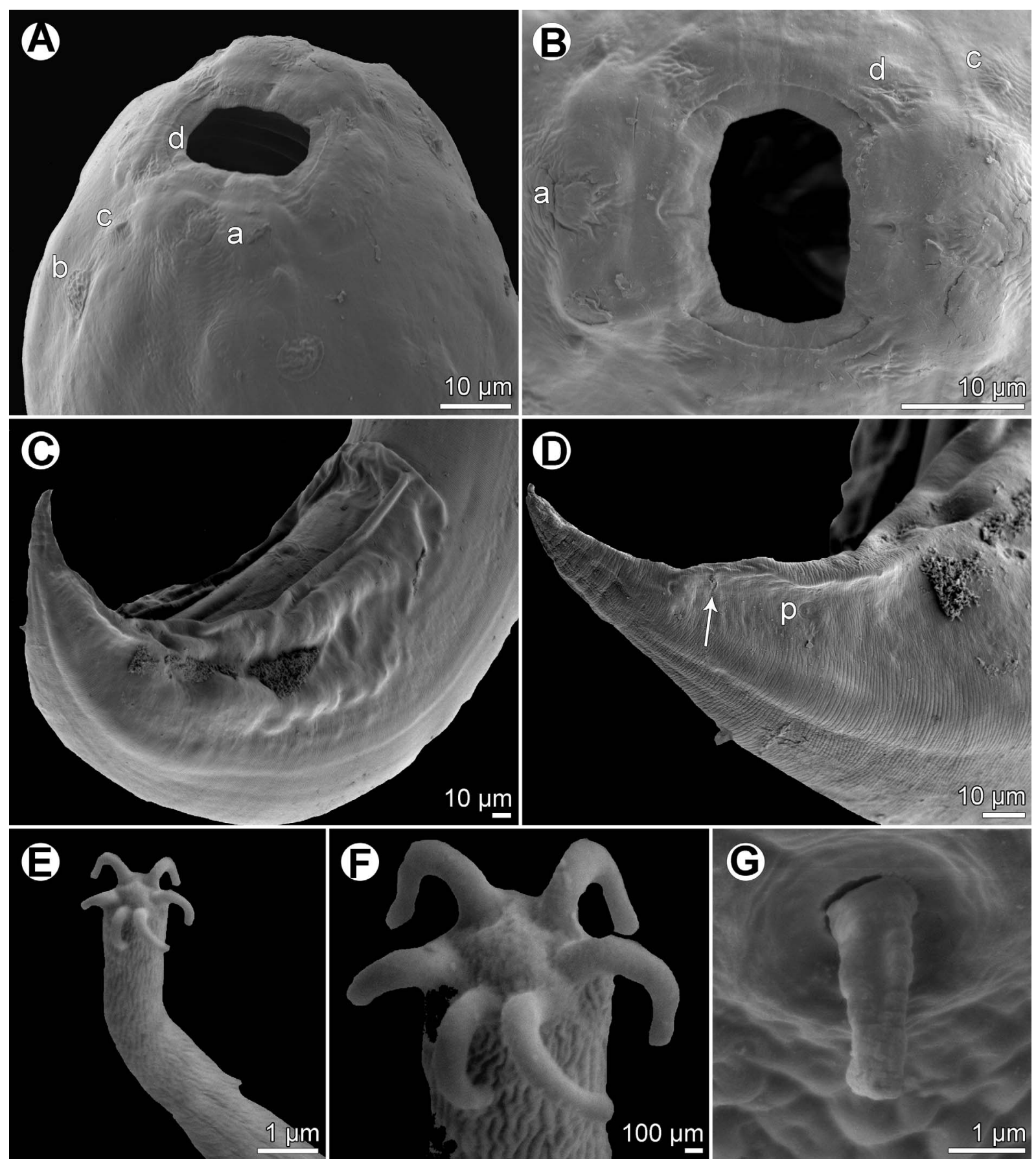

Figure 6. Procamallanus (Spirocamallanus) hexophtalmatis n. sp., scanning electron micrographs. (A, B) Cephalic end of female, sublateral and apical views, respectively; (C) posterior end of male, sublateral view; (D) posterior portion of male tail, lateral view (arrow indicates postanal papilla); (E) distal end of tail of first-stage larva; (F) digital appendages on tail tip of first-stage larva; (G) deirid. (a) amphid; (b) cephalic papilla of external circle; (c) cephalic papilla of middle circle; (d) cephalic papilla of internal circle; (p) phasmid.

of Sciences, České Budějovice, Czech Republic (male holotype and female allotype, both mounted on SEM stub, N-1202); Muséum National d'Histoire Naturelle, Paris, France (two paratypes, MNHN JNC 2736).

Etymology: The specific name of this nematode relates to the genitive form of the species name of the host.

\section{Description}

General: Medium-sized nematode with finely transversely striated cuticle. Mouth aperture oval, surrounded by 12 submedian cephalic papillae arranged in three circles, each formed by four papillae; papillae of outer circle distinctly larger; each of 
four small inner papillae present near margin of oral aperture accompanied by distinct proximal pore; pair of small lateral amphids present (Figs. 5D, 6A and 6B). Buccal capsule orange, thick-walled, longer than wide, with simple, well-developed basal ring. Maximum width/length ratio of buccal capsule 1:1.25-1.27. Inner surface of capsule provided with 11-12 spiral ridges in lateral view, of which three incomplete (Figs. 5B, 5C and 5E). Muscular oesophagus shorter than glandular oesophagus; both parts of oesophagus somewhat expanded near their posterior ends (Fig. 5A). Intestine brown, narrow. Deirids small, simple, with rounded end situated just anterior to level of nerve ring (Figs. 5A, 5B, 5E, 5K and 6G). Excretory pore located at level of junction of both parts of oesophagus (Fig. 5A).

Male (one complete specimen, holotype; measurements of one incomplete specimen in parentheses): Length of body 15.52 (body with missing posterior end 10.74) $\mathrm{mm}$, maximum width 272 (231). Buccal capsule including basal ring 84 (75) long, its width 66 (60); basal ring 9 (6) long and 42 (39) wide. Maximum width/length ratio of buccal capsule 1:1.27 (1:1.25). Spiral ridges 12 (11) in number, 3 (3) incomplete. Length of muscular oesophagus 394 (354), maximum width 81 (69); length of glandular oesophagus 598 (558), maximum width 111 (72); length ratio of muscular and glandular oesophagus 1:1.52 (1:1.58). Length of entire oesophagus and buccal capsule representing $7(-) \%$ of body length. Deirids, nerve ring and excretory pore 222 (219), 258 (245) and 503 (435) from anterior extremity, respectively. Posterior end of body ventrally bent, provided with wide, vesiculated caudal alae supported by pedunculate papillae; anteriorly alae interconnected by mound, forming a kind of pseudosucker, and posteriorly reaching to caudal terminal spines (Figs. 5F, 5G, 6C and 6D). Preanal papillae: three pairs of subventral pedunculate papillae, of which second and third pairs closer to each other than first and second pairs; postanal papillae: six pairs of pedunculate papillae, four subventral and two lateral (last pair representing phasmids); additional two pairs of small, transversely-elongate sessile ventral papillae surrounding cloacal opening (Figs. 5F and $5 \mathrm{G})$. Spicules unequal, with sharply pointed distal ends; large (right) spicule 324 (-) long; small (left) spicule less sclerotized, $180(-)$ long (Fig. 5F). Length ratio of spicules 1:1.80 (-). Gubernaculum absent. Tail conical, 164 (-) long, with two (dorsal and ventral) small terminal cuticular spines 3 (-) long (Figs. 5F and 5G).

Female (one larvigerous specimen, allotype; measurements of one incomplete ovigerous specimen in parentheses): Length of body 24.00 (body with missing anterior end 14.31) mm, maximum width 571 (394). Buccal capsule including basal ring 99 (-) long and $78(-)$ wide; basal ring $9(-)$ long and $54(-)$ wide. Maximum width/length ratio of buccal capsule 1:1.27 (-). Number of spiral ridges 11 (-). Length of muscular oesophagus $476(-)$, maximum width $108(-)$; length of glandular oesophagus $816(-)$, maximum width $138(-)$; length ratio of muscular and glandular oesophagus 1:1.71 (-). Length of entire oesophagus and buccal capsule representing $6(-) \%$ of body length. Deirids, nerve ring and excretory pore 299 (-), $313(-)$ and $585(-)$, respectively, from anterior extremity. Vulva pre-equatorial, $11.70(-) \mathrm{mm}$ from anterior extremity, at $49(-) \%$ of body length. Vulval lips not elevated. Vagina directed anteriorly from vulva. Uterus filled with numerous larvae 444-480 long and six in maximum width with slender tail (uterus with eggs); tail tip of larvae provided with six digital processes (Figs. 6E and 6F). Female tail broad, rounded, its posterior end abruptly narrowed to form digit-like protrusion provided with 2 (2), dorsal and ventral, small terminal cuticular spikes; length of entire tail 82 (58); digit-like protrusion 39 (21) long, 15 (12) wide, length of spines 3 (3) (Figs. 5H, 5I and 5J).

\section{Remarks}

As with the previous species, $P$. ( $S$.) hexophtalmatis n. sp. also belongs to the morphological group of species of the subgenus Spirocamallanus characterized by the presence of wide caudal alae, three pairs of pedunculate preanal papillae, two unequal spicules and two caudal spikes on a digital projection in the female (see above). Of the species of this group occurring in the Indo-Pacific region, based on the length of the right spicule, $P$. hexophtalmatis $\mathrm{n}$. $\mathrm{sp}$. is similar to $P$. anguillae $(289-384 \mu \mathrm{m}$ vs $324 \mu \mathrm{m})$, P. gobiomori $(318-348 \mu \mathrm{m})$, P. guttatusi (204$350 \mu \mathrm{m}), P$. istiblenni $(263-302 \mu \mathrm{m}), P$. monotaxis $(279$ $315 \mu \mathrm{m}), \quad P$. rigbyi $(315-360 \mu \mathrm{m})$ and $P$. variolae (327-357 $\mu \mathrm{m})[17,22,25,26,29-31,33,35,40,46]$.

However, all these species, except for $P$. variolae, have deirids situated near the mid-point between the base of the buccal capsule and the nerve ring ( $v s$ deirids situated just anterior to the nerve ring). Based on this feature, the new species resembles only $P$. variolae, in which, however, the deirids are located slightly posterior ( $v s$ anterior) to the level of the nerve ring. Although $P$. variolae and $P$. hexophtalmatis n. sp. have the same numbers (11-12) of spiral ridges in the buccal capsule and the body lengths of their gravid (larvigerous) females are identical (approximately $24 \mathrm{~mm}$ ), the new species differs from $P$. variolae in the length ratio of the muscular and glandular portions of the oesophagus (1:1.5-1.6 in males and 1:1.7 in the gravid female vs 1:1.1-1.3 in males and $1: 1.3$ in the gravid female), location of the excretory pore at the level of the muscular and glandular oesophageal junction ( $v s$ somewhat posterior to this junction) and in that the vagina of the gravid female is directed anteriorly (vs posteriorly) from the vulva. Whereas the tail of both gravid and subgravid females in the new species is widely rounded (Figs. 5H and 5I), that of the gravid female of $P$. variolae is somewhat more conical. Moreover, both these species differ in the family of their fish hosts (Pinguipedidae vs Serranidae).

In addition to a different location of deirids (see above), $P$. anguillae, $P$. istiblenni, $P$. monotaxis and $P$. rigbyi usually have more numerous spiral ridges in the buccal capsule $(10-15,12-15,10-17$ and 13-14, respectively vs 11-12), whereas the ridges of $P$. gobiomori are less numerous (8-10). These five species also differ from $P$. hexophtalmatis n. sp. in the family of their hosts (Anguillidae, Blenniidae, Lethrinidae, Sciaenidae and Eleotridae, respectively vs Pinguipedidae).

Moravec et al. [27] reported Procamallanus (Spirocamallanus) sp. 1 from P. hexophtalma in New Caledonia. However, although the general morphology of the only available specimen (subgravid female) was similar to that of $P$. hexophtalmatis n. sp., the spiral ridges in its buccal capsule were more numerous (16), deirids were located approximately at $2 / 3$ of a distance between the base of the buccal capsule and the nerve ring and 


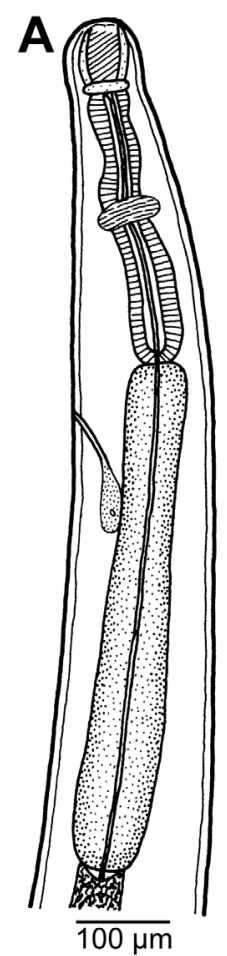

H

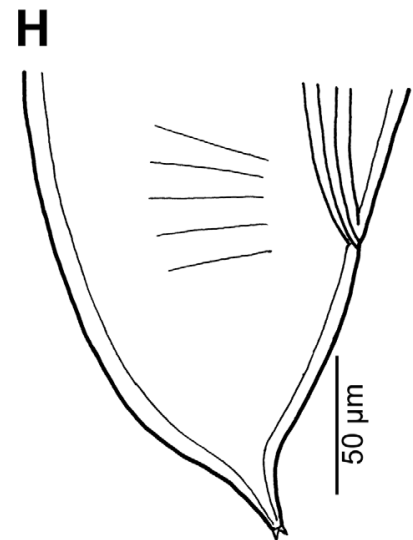

B

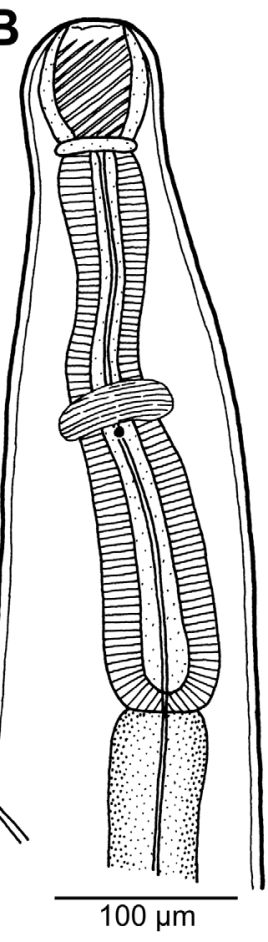

C

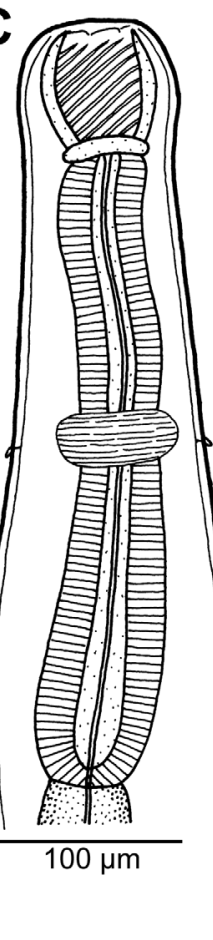

D

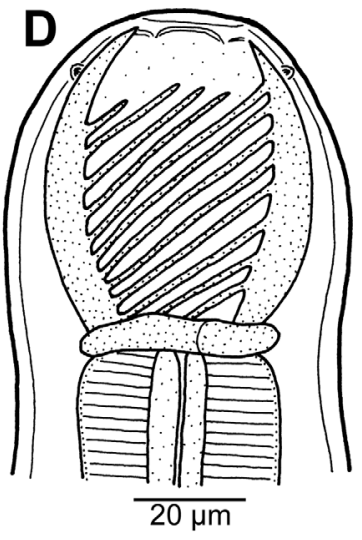

$\mathbf{F}$

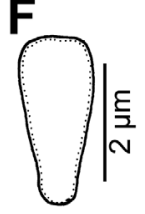

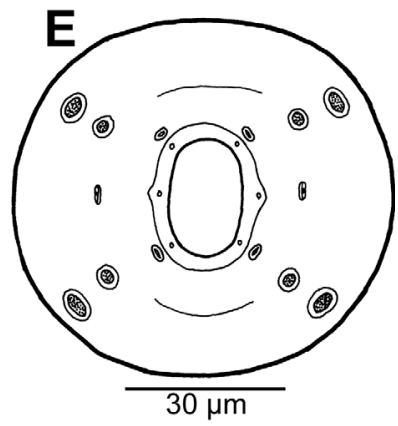
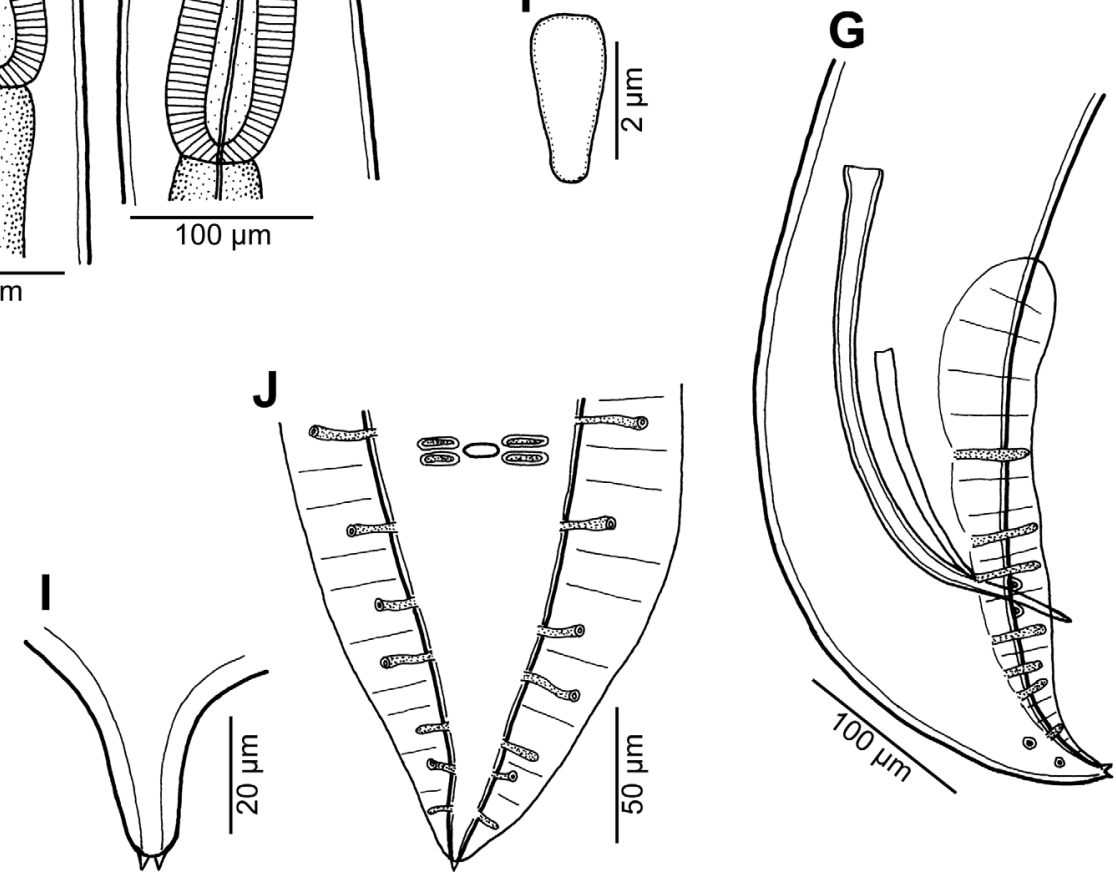

Figure 7. Procamallanus (Spirocamallanus) synodi n. sp. from Synodus dermatogenys. (A) Anterior end of male, lateral view; (B) same, larger magnification; (C) anterior end of female, dorsoventral view; (D) buccal capsule of male, lateral view; (E) cephalic end, apical view; (F) deirid; (G) posterior end of male, lateral view; (H) tail of female, lateral view; (I) tail tip of female, lateral view; (J) tail of male, ventral view.

its tail was more conical as compared with that of the subgravid female of $P$. hexophtalmatis, resembling thus $P$. monotaxis [27]. Therefore, allocation of this specimen to P. hexophtalmatis is uncertain. Rigby and Adamson [39] reported P. monotaxis, originally described from a lethrinid fish from Hawaii [35], from Parapercis millepunctata (Günther) and members of several other fish families in French Polynesia, but this identification needs to be confirmed. In New Caledonia, $P$. monotaxis was recorded only from Lethrinus spp. [25].

The SEM examination of the first-stage larva of $P$. hexophtalmatis shows that the tail tip bears six digitiform processes (Figs. 6E and 6F). Similar caudal processes were previously observed in first-stage larvae of Camallanus cotti and C. lacustris [24] and those of two Procamallanus species from African freshwater fishes [18]. Apparently, these caudal processes serve the larva to better attach by its tail to the bottom, after the larvae are released into the water [24].

Procamallanus (Spirocamallanus) synodi $\mathrm{n} . \mathrm{sp}$. Figures 7, 8

urn:lsid:zoobank.org:act:0F58977A-B15C-4888-ABD1245B4016032E

Type host: Sand lizardfish Synodus dermatogenys Fowler (Synodontidae, Aulopiformes).

Site of infection: Intestine.

Type locality: Near Récif Toombo, off Nouméa, New Caledonia, $22^{\circ} 32^{\prime} 583 \mathrm{~S}, 166^{\circ} 28^{\prime} 978 \mathrm{E}$ (collected 5 November 2008). 


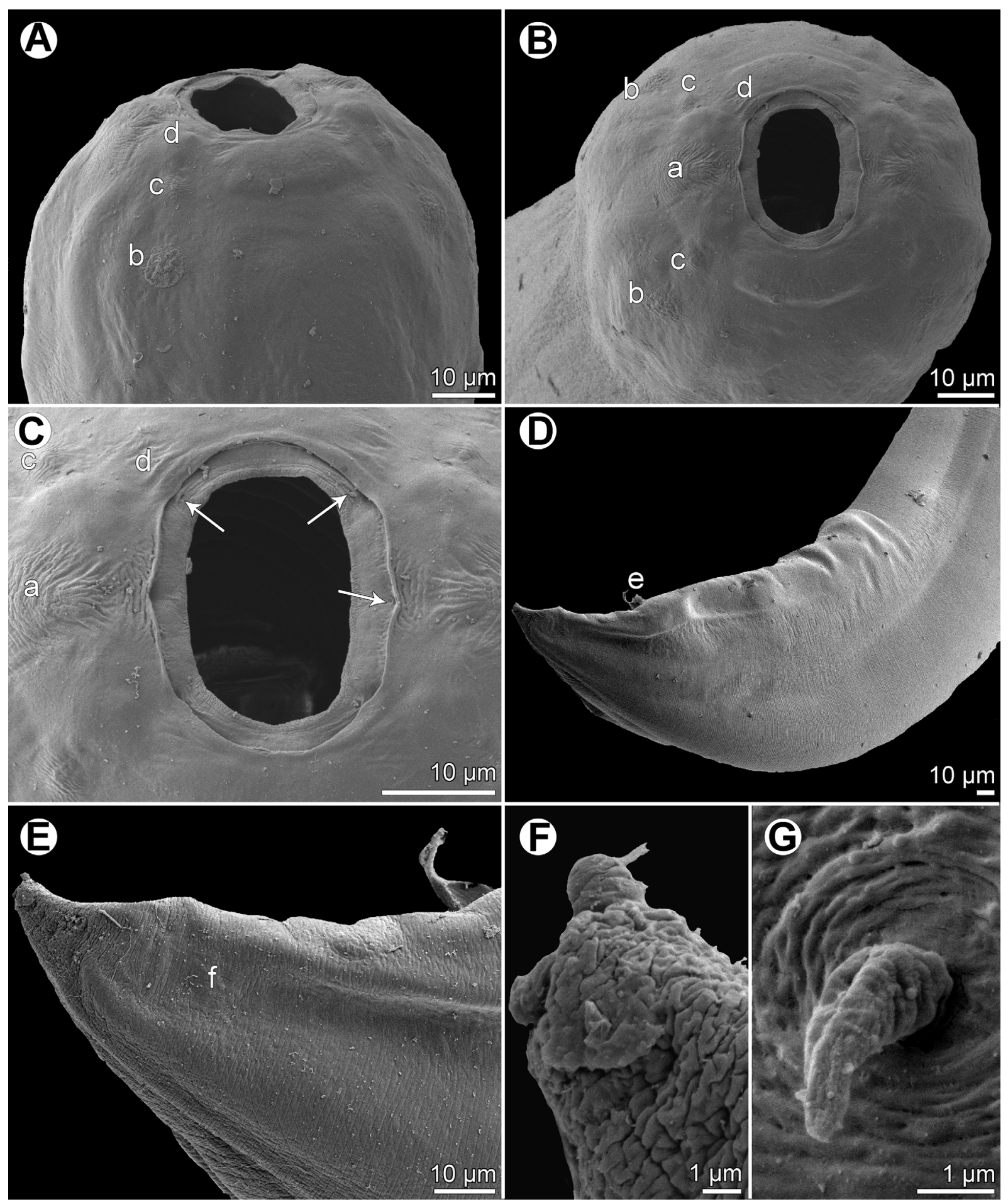

Figure 8. Procamallanus (Spirocamallanus) synodi n. sp., scanning electron micrographs. (A, B) Cephalic end, subdorsoventral and apical views, respectively; (C) region of oral aperture, apical view (arrows indicate circumoral pores); (D) posterior end of male, lateral view; (E) tail of male, lateral view; (F) tail tip of female, lateral view; (G) deirid. (a) amphid; (b) cephalic papilla of external circle; (c) cephalic papilla of middle circle; (d) cephalic papilla of internal circle; (e) cloaca; (f) phasmid.

Prevalence, intensity and details about fish: 1 fish infected/4 fish examined; 5 nematodes. The infected fish (Fish number JNC2756) was $120 \mathrm{~mm}$ in fork length and $20 \mathrm{~g}$ in weight.
Deposition of type specimens: Helminthological Collection, Institute of Parasitology, Biology Centre of the Czech Academy of Sciences, České Budějovice, Czech Republic (male holotype and female allotype, both mounted on SEM stub, N-1203); 
Muséum National d'Histoire Naturelle, Paris, France (three paratypes, MNHN JNC 2756A).

Etymology: The specific name of this nematode relates to the genitive form of the generic name of the host.

\section{Description}

General: Medium-sized nematode with finely transversely striated cuticle. Mouth aperture oval, surrounded by 12 submedian cephalic papillae arranged in three circles, each formed by four papillae; papillae of outer circle distinctly larger; each of four small inner papillae present near margin of oral aperture accompanied by distinct proximal pore; pair of small lateral amphids present (Figs. 7E, 8A, 8B and 8C). Buccal capsule orange, thick-walled, longer than wide, with simple, welldeveloped basal ring. Maximum width/length ratio of buccal capsule 1:1.00-1.23. Inner surface of capsule provided with 10-12 spiral ridges in lateral view, 3-5 of them being incomplete (Figs. 7B, 7C and 7D). Muscular oesophagus shorter than glandular oesophagus; both parts of oesophagus slightly expanded near their posterior ends (Figs. 7A, 7B and 7C). Intestine brown, narrow. Deirids small, simple, with rounded end situated at level of nerve ring or just posterior to it (Figs. 7B, 7C, 7F and 8G). Excretory pore located short distance posterior to anterior end of glandular oesophagus (Fig. 7A).

Male (two specimens; holotype; measurements of paratype in parentheses): Length of body 9.38 (9.13) mm, maximum width 231 (218). Buccal capsule including basal ring 66 (81) long, its width 60 (66); basal ring 6 (9) long and 39 (48) wide. Maximum width/length ratio of buccal capsule 1:1.10 (1:1.23). Spiral ridges 12 (12), 0 (5) of which incomplete. Length of muscular oesophagus 354 (340), maximum width 63 (69); length of glandular oesophagus 462 (639), maximum width 78 (93); length ratio of muscular and glandular oesophagus 1:1.31 (1:1.88). Length of entire oesophagus and buccal capsule representing 9 (12)\% of body length. Deirids, nerve ring and excretory pore 279 (246), 245 (231) and 462 (476), respectively, from anterior extremity. Posterior end of body ventrally bent, provided with wide, vesiculated caudal alae supported by pedunculate papillae; anteriorly alae interconnected by mound, forming a kind of pseudosucker, and posteriorly reaching to caudal terminal spines (Figs. 7G, 7J and 8D). Preanal papillae: three pairs of subventral pedunculate papillae, of which second and third pairs closer to each other than first and second pairs; postanal papillae: six pairs of pedunculate papillae, four subventral and two lateral (last pair representing phasmids); additional two pairs of small, transversely-elongate sessile ventral papillae surrounding cloacal opening (Figs. 7G, 7J and $8 \mathrm{E}$ ). Spicules unequal, with sharply pointed distal ends (Fig. 7G); large (right) spicule 225 (330) long; small (left) spicule less sclerotized, 204 (147) long. Length ratio of spicules 1:1.10 (1:2.24). Gubernaculum absent. Tail conical, 123 (126) long, with two (dorsal and ventral) small terminal cuticular spines 3 (3) long (Fig. 7G).

Female (two ovigerous specimens; allotype; measurements of paratype in parentheses. Measurements of one nongravid specimen in brackets): Length of body 12.10 (11.74) [7.05] mm, maximum width 286 (218) [190]. Buccal capsule including basal ring 75 (66) [60] long and 72 (66) [51] wide; basal ring 9 (9) [6] long and 45 (39) [39] wide. Maximum width/length ratio of buccal capsule 1:1.04 (1:1.00) [1:1.18]. Number of spiral ridges 11 (11) [10], of which 4 (3) [3] incomplete. Length of muscular oesophagus 394 (422) [286], maximum width 75 (81) [54]; length of glandular oesophagus 530 (666) [394], maximum width 90 (111) [66]; length ratio of muscular and glandular oesophagus 1:1.35 (1:1.58) [1:1.38]. Length of entire oesophagus and buccal capsule representing 5 (10) [11]\% of body length. Deirids, nerve ring and excretory pore 285 (285) [219], 245 (272) [190] and 544 (598) [408], respectively, from anterior extremity. Vulva equatorial or somewhat postequatorial, 6.03 (6.19) [3.86] $\mathrm{mm}$ from anterior extremity, at 50 (53) [55]\% of body length. Vulval lips not elevated. Vagina directed posteriorly from vulva. Uterus filled with eggs (with eggs) [without eggs]. Tail broad, conical, its posterior end narrowed to form narrow conical protrusion provided with 2 (2) [2], dorsal and ventral, small terminal cuticular spikes; length of entire tail 171 (114) [96]; protrusion 24 (27) [45] long, 15 (15) [15] wide, length of spines 3 (3) [3] (Figs. 7H, 7I, 8F).

\section{Remarks}

The present nematodes belong to the same morphological group of Procamallanus (Spirocamallanus) as the previous two species, $P$. bothi n. sp. and P. hexophtalmatis n. sp. By the length of the right spicule, they resemble nine very similar species occurring in the Indo-Pacific region, viz. P. anguillae, $P$. bothi n. sp., P. gobiomori, $P$. hexophtalmatis n. sp., $P$. guttatusi, $P$. istiblenni, $P$. monotaxis, $P$. rigbyi and $P$. variolae (see above). However, in having deirids located at or near the level of the nerve ring, they resemble only $P$. hexophtalmae and P. variolae, whereas deirids in other species are situated approximately in the mid-point between the buccal capsule and the nerve ring (in P. istiblenni in $2 / 3$ of this distance).

In contrast to the new species, the female tail of $P$. hexophtalmatis n. sp. is widely rounded ( $v s$ conical), deirids are located slightly anterior to the level of the nerve ring ( $v s$ deirids at or just posterior to this level), the excretory pore is at the level of the junction of both parts of the oesophagus ( $v s$ at a short distance posterior to the anterior end of the glandular oesophagus), the vulva is slightly pre-equatorial (vs equatorial or somewhat postequatorial), the vagina is directed anteriorly ( $v s$ posteriorly) from the vulva and the males and females are distinctly longer (male $15.5 \mathrm{~mm}$, gravid female $24.0 \mathrm{~mm}$ vs males $9.1-9.4 \mathrm{~mm}$, subgravid females 11.7-12.1 mm).

Procamallanus $(S$.) variolae differs from the new species in the shape of the female tail (rounded $v s$ conical), in having a distinctly pre-equatorial vulva ( $v s$ vulva equatorial or postequatorial), a largely different length ratio of the muscular and glandular parts of the oesophagus (1:1.1-1.3 vs 1:1.3-1.9) and in that the males and females of this species are longer (males $10.5-12.7 \mathrm{~mm}$, gravid female $24.5 \mathrm{~mm}$ vs males $9.1-9.4 \mathrm{~mm}$ and subgravid females $11.7-12.10 \mathrm{~mm}$ ); the buccal capsule of $P$. variolae is larger $(84-87 \times 60-66 \mu \mathrm{m}$ in males and 

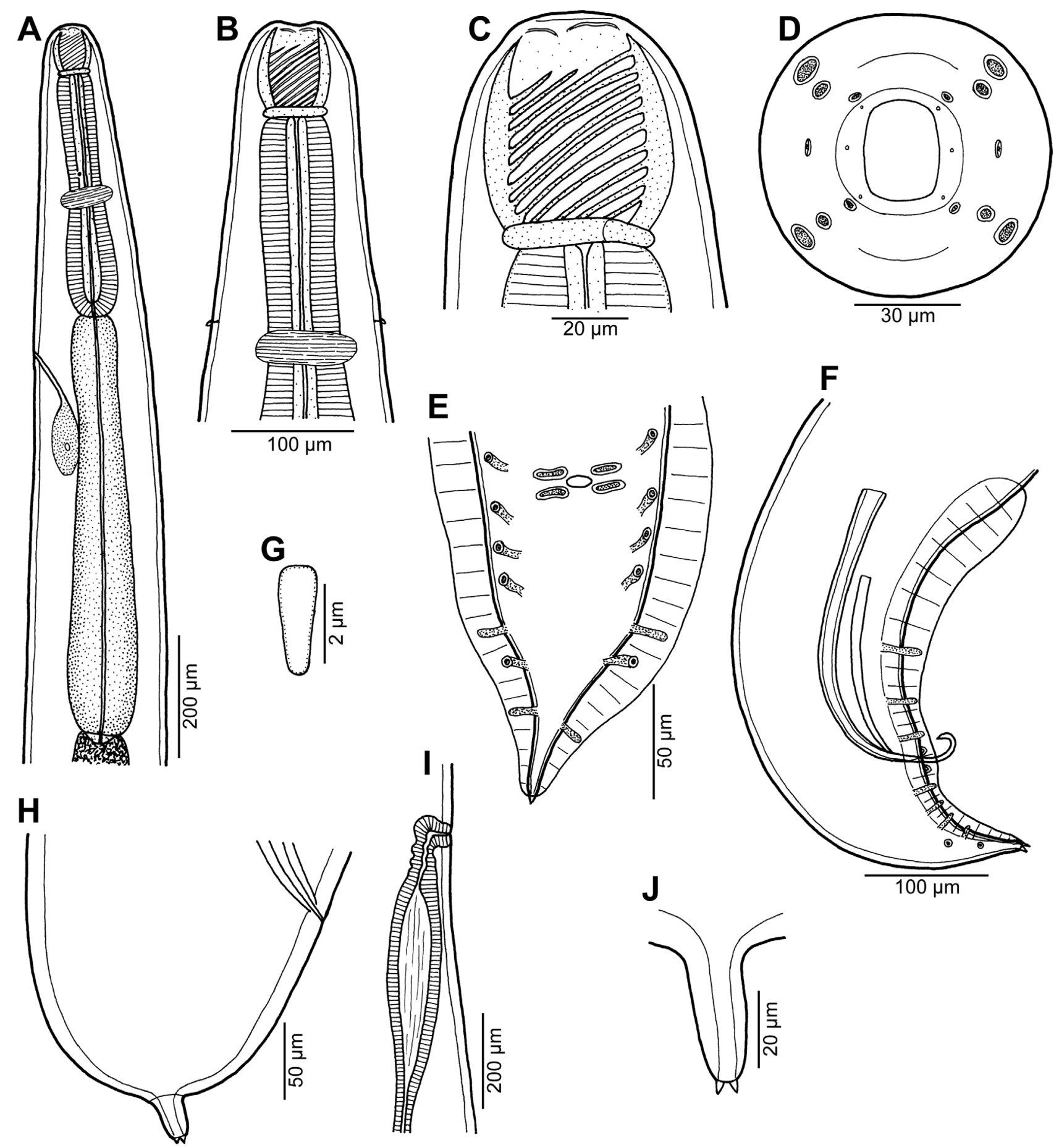

Figure 9. Procamallanus (Spirocamallanus) thalassomatis n. sp. from Thalassoma lutescens. (A, B) Anterior end of male, lateral and dorsoventral views, respectively; (C) buccal capsule of male, lateral view; (D) cephalic end, apical view; (E) tail of male, ventral view; (F) posterior end of male, lateral view; $(\mathrm{G})$ deirid; $(\mathrm{H})$ tail of female, lateral view; (I) vulva and distal end of vagina, lateral view; (J) tail tip of female, lateral view.

$99 \times 78 \mu \mathrm{m}$ in female $v s 66-81 \mu \mathrm{m}$ in males and 66-72 $\times 66-$ $72 \mu \mathrm{m}$ in subgravid females). Moreover, the hosts of both $P$. hexophtalmatis and $P$. variolae belong to other fish families than that of the new species (Pinguipedidae and Serranidae, respectively vs Synodontidae).

Procamallanus $(S$.) synodi n. sp. is the first species of this genus reported from a fish of the family Synodontidae.

\section{Procamallanus (Spirocamallanus) thalassomatis} n. sp. Figures 9-11

urn:lsid:zoobank.org:act:1B89617C-7328-471A-9A60B25190D68E0E

Type host: Yellow-brown wrasse Thalassoma lutescens (Lay et Bennett) (Labridae, Perciformes). 


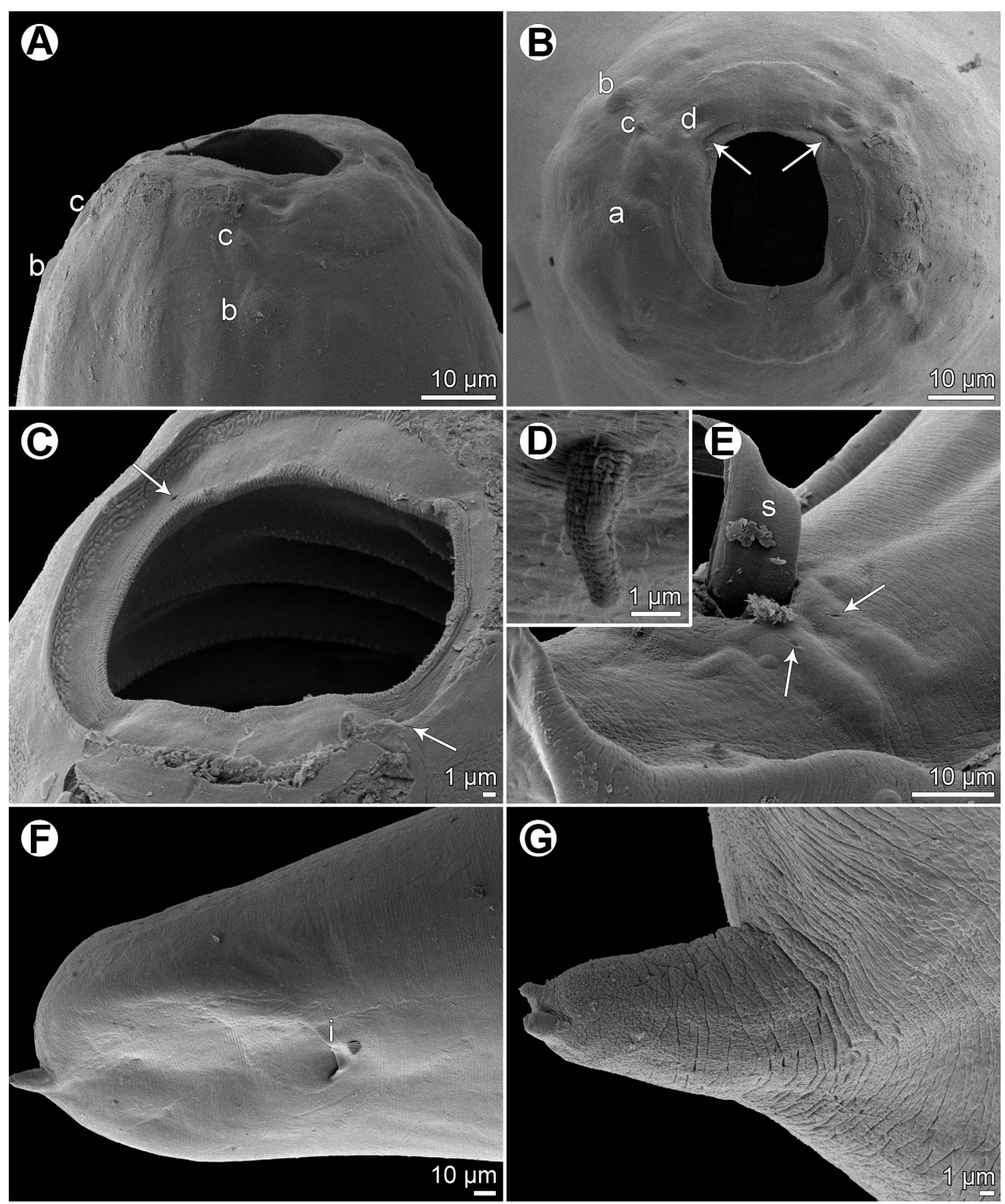

Figure 10. Procamallanus (Spirocamallanus) thalassomatis n. sp., scanning electron micrographs. (A, B) Cephalic end, sublateral and apical views, respectively (arrows indicate circumoral pores); (C) region of oral aperture, subapical view (arrows indicate circumoral pores); (D) deirid; (E) region of cloaca, sublateral view (arrows indicate pores of flat papillae around cloaca); (F) tail of gravid female, ventral view; (G) caudal projection of gravid female, sublateral view. (a) amphid; (b) cephalic papilla of external circle; (c) cephalic papilla of middle circle; (d) cephalic papilla of internal circle; (i) anus; (s) spicule.

Site of infection: Intestine.

Type locality: Reef near Passe de Dumbéa, New Caledonia, $22^{\circ} 21^{\prime} 189 \mathrm{~S}, 166^{\circ} 15^{\prime} 158 \mathrm{E}$ (collected 16 October 2009).
Prevalence, intensity and details about fish: 1 fish infected/8 fish examined; 3 nematodes. The infected fish (Fish number: JNC3074) was $173 \mathrm{~mm}$ in fork length and $63 \mathrm{~g}$ in weight. 


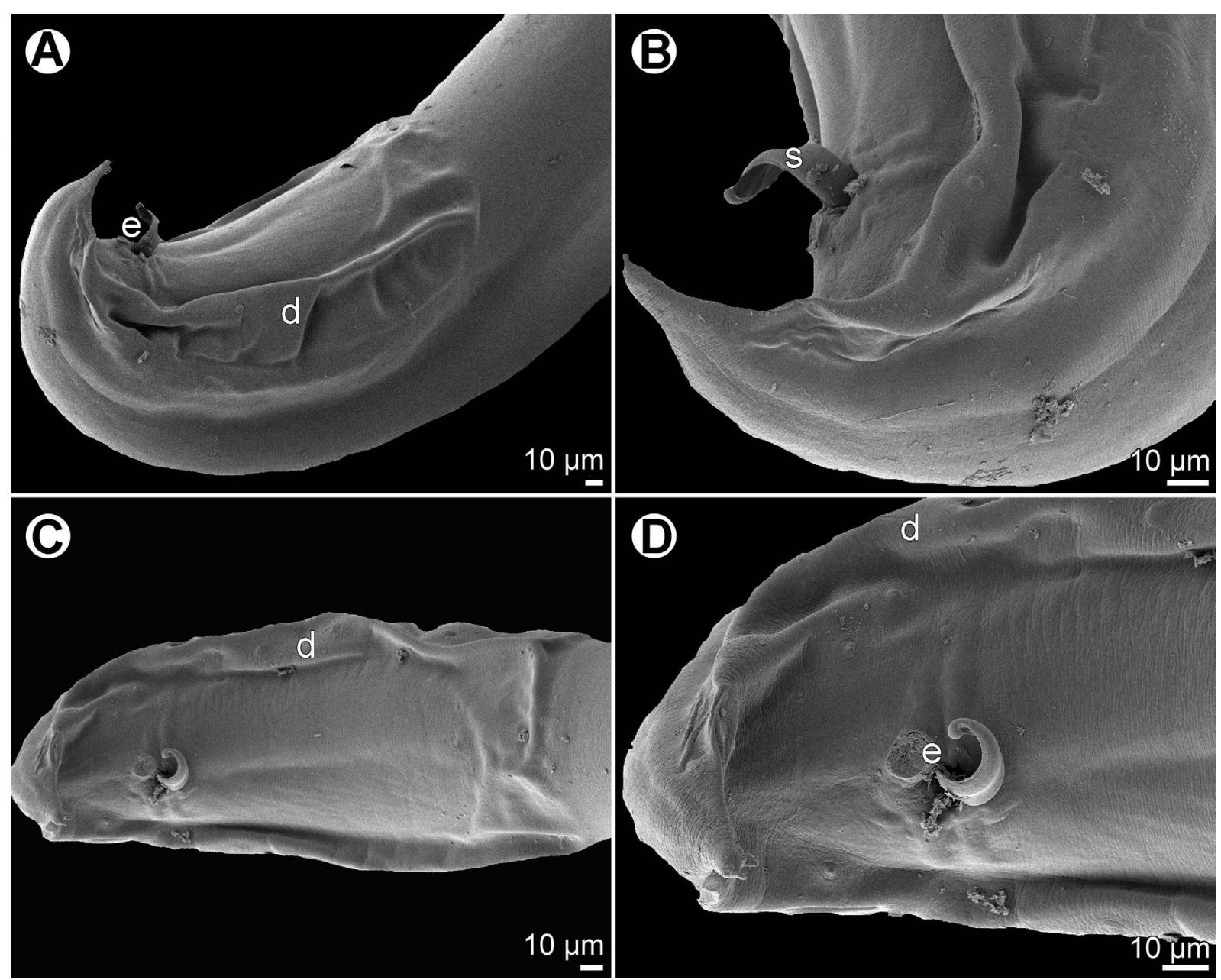

Figure 11. Procamallanus (Spirocamallanus) thalassomatis n. sp., scanning electron micrographs of male. (A) Posterior end of body, lateral view; (B) tail, lateral view; (C) posterior end of body, ventral view; (D) tail, ventral view. (d) caudal ala; (e) cloaca; (s) spicule.

Deposition of type specimens: Helminthological Collection, Institute of Parasitology, Biology Centre of the Czech Academy of Sciences, České Budějovice, Czech Republic (male holotype and female allotype, both mounted on SEM stub, N-1204); Muséum National d'Histoire Naturelle, Paris, France (female paratype, MNHN JNC 3074).

Etymology: The specific name of this nematode relates to the genitive form of the generic name of the host.

\section{Description}

General: Medium-sized nematode with finely transversely striated cuticle. Mouth aperture oval, surrounded by 12 submedian cephalic papillae arranged in three circles, each formed by four papillae; papillae of outer circle larger; each of four small inner papillae present near margin of oral aperture accompanied by distinct proximal pore; pair of small lateral amphids present (Figs. 9D, 10A, 10B and 10C). Buccal capsule orange, thickwalled, longer than wide, with simple, well-developed basal ring. Maximum width/length ratio of buccal capsule $1: 1.07-1.21$. Inner surface of capsule provided with 11-12 spiral ridges in lateral view, 4-5 of them being incomplete (Figs. 9B, 9C and 10C). Muscular oesophagus shorter than glandular oesophagus; both parts of oesophagus slightly expanded near their posterior ends (Fig. 9A). Intestine brown, narrow. Deirids small, simple, with rounded end situated slightly anterior to level of nerve ring (Figs. 9B, 9G and 10D). Excretory pore located somewhat posterior to anterior end of glandular oesophagus (Fig. 9A).

Male (one specimen, holotype): Length of body $12.53 \mathrm{~mm}$, maximum width 313 . Buccal capsule including basal ring 87 long, its width 72; basal ring 12 long and 57 wide. Maximum width/length ratio of buccal capsule 1:1.21. Spiral ridges 12,5 of which incomplete. Length of muscular oesophagus 435, maximum width 93; length of glandular oesophagus 748, maximum width 126; length ratio of muscular and glandular oesophagus 1:1.72. Length of entire oesophagus and buccal capsule representing $10 \%$ of body length. Deirids, nerve ring and excretory pore 279, 299 and 558, respectively, from anterior extremity. Posterior end of body ventrally bent, provided with wide, vesiculated caudal alae supported by pedunculate papillae; anteriorly alae interconnected by mound, forming a kind of pseudosucker, and posteriorly reaching to caudal terminal spines (Figs. 9E, 9F, 11A, 11B, 11C and 11D). Preanal papillae: three pairs of subventral pedunculate papillae, of which second and third pairs closer to each other than first 
and second pairs; postanal papillae: six pairs of pedunculate papillae, four subventral and two lateral (last pair representing phasmids); additional two pairs of small, transversely-elongate sessile ventral papillae surrounding cloacal opening (Figs. 9E, 9F, 10E, 11A, 11B and 11D). Spicules unequal, with sharply pointed distal ends (Fig. 9F); large (right) spicule 330 long; small (left) spicule less sclerotized, 144 long. Length ratio of spicules 1:1.48 (1:2.29). Gubernaculum absent. Tail conical, 141 long, with two (dorsal and ventral) small terminal cuticular spines four long (Figs. 9F and 11A).

Female (two larvigerous specimens; allotype; measurements of paratype in parentheses): Length of body 25.55 (25.66) mm, maximum width 639 (598). Buccal capsule including basal ring 96 (99) long and 90 (90) wide; basal ring 12 (12) long and 63 (63) wide. Maximum width/length ratio of buccal capsule 1:1.07 (1:1.10). Number of spiral ridges 11 (12), of which 4 (5) incomplete. Length of muscular oesophagus 625 (666), maximum width 136 (122); length of glandular oesophagus 1088 (1170), maximum width 177 (177); length ratio of muscular and glandular oesophagus 1:1.74 (1:1.76). Length of entire oesophagus and buccal capsule representing 7 (8)\% of body length. Deirids, nerve ring and excretory pore 313 (326), 381 (367) and 762 (768), respectively, from anterior extremity. Vulva slightly pre-equatorial (equatorial), 12.65 (12.92) $\mathrm{mm}$ from anterior extremity, at 49 (50)\% of body length. Vulval lips not elevated (Fig. 9I). Vagina directed posteriorly from vulva. Uterus filled with numerous larvae 381-396 long, with slender tail. Female tail broad, rounded, its posterior end abruptly narrowed to form digit-like protrusion provided with 2 (2), dorsal and ventral, small terminal cuticular spikes; length of entire tail 195 (201); digit-like protrusion 30 (42) long, 15 (15) wide, length of spines 3 (3) (Figs. 9H, 9J, 10F and 10G).

\section{Remarks}

The nematodes from $T$. lutescens belong to the same morphological group of Procamallanus (Spirocamallanus) as the species $P$. bothi n. sp., $P$. hexophtalmatis n. sp. and $P$. synodi n. sp. (see above). From the Indo-Pacific species of this group, $P$. pereirai and $P$. similis can be differentiated from $P$. thalassomatis $\mathrm{n}$. sp. by possessing a distinctly longer right spicule $(430 \mu \mathrm{m}$ and $435-492 \mu \mathrm{m}$, respectively vs $330 \mu \mathrm{m})$ and $P$. bothi in having a shorter right spicule $(267-270 \mu \mathrm{m})$, whereas the length of this spicule in the remaining species (P. anguillae, P. gobiomori, P. guttatusi, P. istioblenni, $P$. monotaxis, $P$. rigbyi and $P$. variolae) is rather similar. However, in having deirids located near the level of the nerve ring, they resemble only $P$. hexophtalmatis, $P$. synodi and $P$. variolae, whereas deirids in other species are situated approximately in the mid-way between the buccal capsule and the nerve ring (in $P$. istiblenni in $2 / 3$ of this distance).

On the basis of the location of deirids somewhat anterior to the level of the nerve ring, $P$. thalassomatis n. sp. resembles $P$. hexophtalmatis n. sp., whereas deirids in $P$. variolae and $P$. synodi $\mathrm{n}$. sp. are located at the level of the nerve ring or just posterior to this level. However, $P$. thalassomatis differs from $P$. hexophtalmatis in the vagina directed anteriorly ( $v s$ posteriorly) from the vulva; although the male body of the former species is shorter than that of the latter species (12.5 mm vs $15.5 \mathrm{~mm}$ ), its buccal capsule is distinctly larger $(87 \times 72 \mu \mathrm{m}$ vs $75-84 \times 60 \mu \mathrm{m})$. The new species can be differentiated from $P$. variolae mainly by the length ratio of the muscular and glandular parts of the oesophagus (1:1.7-1.8 vs $1: 1.1-1.3)$ and by the percentage of the length of the oesophagus and buccal capsule of the entire body length of gravid females (7-8\% vs 5\%), whereas from $P$. synodi mainly by the shape of the female tail (broadly rounded $v s$ conical) and the larger buccal capsule $(87 \times 72 \mu \mathrm{m}$ in male and $96-99 \times 90 \mu \mathrm{m}$ in gravid female vs 66-81 $\times$ 60-66 $\mu \mathrm{m}$ in male and 66-75 $\times$ 66-72 $\mu \mathrm{m}$ in subgravid female). Moreover, the hosts of $P$. hexophtalmatis, $P$. synodi and $P$. variolae belong to other fish families than that of the new species (Pinguipedidae, Synodontidae and Serranidae, respectively vs Labridae).

Procamallanus $(S$.) thalassomatis $\mathrm{n}$. sp. is the first species of this genus reported from a fish of the family Labridae.

\section{Procamallanus (Spirocamallanus) sp. 3 of Moravec et al., 2006}

Host: Two-lined monocle bream Scolopsis bilineata (Bloch) (Nemipteridae, Perciformes).

Site of infection: Intestine.

Locality: Near Ilôt Sainte-Marie, off Nouméa, New Caledonia, $22^{\circ} 18^{\prime} \mathrm{S}, 166^{\circ} 27^{\prime} \mathrm{E}$ (collected 4 July 2003 and 5 June 2006).

Prevalence, intensity and details about fish: 2 fish infected/12 fish examined; 1 nematode per fish. The infected fish (Fish numbers: JNC619, JNC1917) were 139-190 mm in fork length and 51-156 $\mathrm{g}$ in weight.

Deposition of voucher specimen: Muséum National d'Histoire Naturelle, Paris, France (1 specimen, MNHN JNC619A).

\section{Remarks}

Based on a single specimen (subgravid female) from S. bilineata off New Caledonia, Moravec et al. [27] described Procamallanus $(S$.) sp. 3, characterized by 13 spiral ridges in the buccal capsule and a broad tail with a short smooth projection. Two available specimens (also subgravid females) of the present material from the same host species, 14.12 and $18.84 \mathrm{~mm}$ long, are morphologically identical with that reported by Moravec et al. [27] and there is no doubt that both these forms belong to the same species. By the shape of the female tail and the absence of terminal spikes, this species is similar to $P$. (S.) dispar n. sp. and a few other congeners (see above). However, since conspecific males remain unknown, the species identification of these nematodes is impossible.

\section{Procamallanus (Spirocamallanus) sp. 1 Figure 12}

Host: Shadow trevally Carangoides dinema Bleeker (Carangidae, Perciformes).

Site of infection: Intestine.

Locality: Fishmarket, Nouméa, New Caledonia (collected 13 March 2009). 

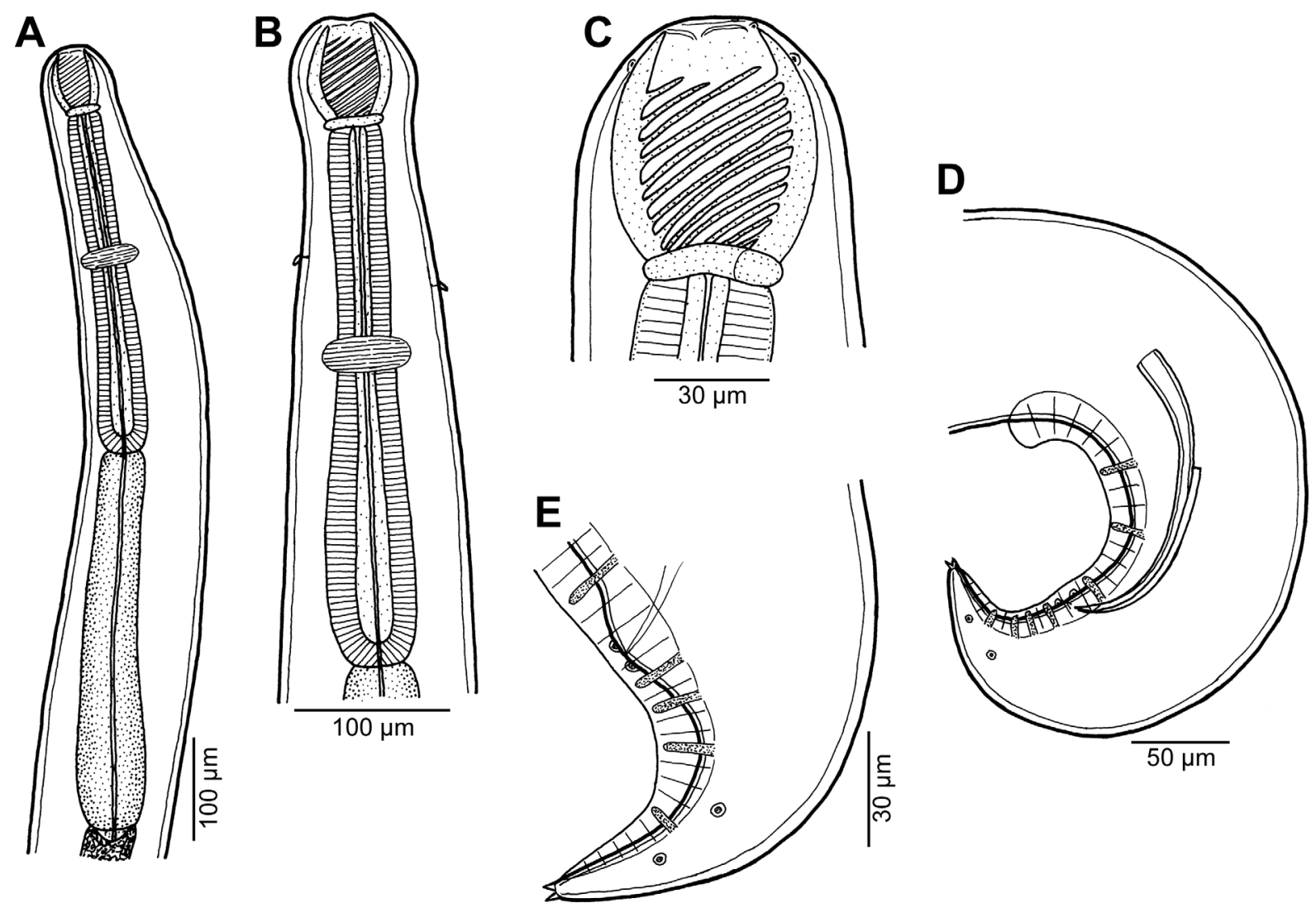

Figure 12. Procamallanus (Spirocamallanus) sp. 1 from Carangoides dinema, male. (A, B) Anterior end, lateral and dorsoventral views, respectively; (C) buccal capsule, lateral view; (D) posterior end of body, lateral view; (E) tail, lateral view.

Prevalence, intensity and details about fish: 1 fish infected/9 fish examined; 1 nematode. The infected fish (Fish number: JNC2882) was $297 \mathrm{~mm}$ in fork length and $577 \mathrm{~g}$ in weight.

Deposition of voucher specimen: Muséum National d'Histoire Naturelle, Paris (MNHN JNC 2882).

\section{Description}

Male (one specimen): Length of body $7.56 \mathrm{~mm}$, maximum width 163. Buccal capsule including basal ring 72 long, its width 60; basal ring 6 long and 39 wide. Maximum width/ length ratio of buccal capsule 1:1.20. Spiral ridges 13, 5 of which incomplete (Fig. 12C). Length of muscular oesophagus 381 , maximum width 60 ; length of glandular oesophagus 449, maximum width 81 (Figs. 12A and 12B); length ratio of muscular and glandular oesophagus 1:1.18. Length of entire oesophagus and buccal capsule representing $12 \%$ of body length. Nerve ring 231 from anterior extremity. Deirids situated somewhat asymmetrically at 165/195 from anterior extremity, slightly posterior to mid-point between base of buccal capsule and nerve ring (Fig. 12B). Excretory pore not located. Posterior end of body ventrally bent, provided with wide, vesiculated caudal alae supported by pedunculate papillae and posteriorly reaching to end of tail. Preanal papillae: three pairs of subventral pedunculate papillae; postanal papillae: six pairs of pedunculate papillae, four subventral and two lateral (last pair representing phasmids); additional two pairs of small, transversely-elongate sessile ventral papillae surrounding cloacal opening (Figs. 12D and 12E). Spicules unequal, with sharply pointed distal ends; right spicule 258 long; small (left) spicule less sclerotized, 198 long (Fig. 12D). Length ratio of spicules 1:1.30. Tail 114 long, with two (dorsal and ventral) small terminal cuticular spines three long (Fig. 12E).

\section{Remarks}

Only a single male specimen of this nematode was available to study. Because some taxonomically important morphological features are found in females in this group of nematodes (e.g., the shape of the female tail), species identification was not possible. No species of Procamallanus has so far been reported from a carangid fish.

\section{Procamallanus (Spirocamallanus) sp. 2 Figure 13}

Host: Zebra shark Stegostoma fasciatum (Hermann) (Stegostomatidae, Orectolobiformes).

Site of infection: Stomach.

Locality: Near Récif Aboré, off Nouméa, New Caledonia, $22^{\circ} 24^{\prime} 060 \mathrm{~S}, 166^{\circ} 18^{\prime} 961 \mathrm{E}$ (collected 3 May 2005).

Prevalence, intensity and detail about fish: 1 fish infected/1 fish examined; 1 nematode; the shark was $2080 \mathrm{~mm}$ in length. Photograph of fish available from: https://commons.wikimedia. org/wiki/File:Stegostoma_fasciatum_JNC1529_Body.JPG 
A
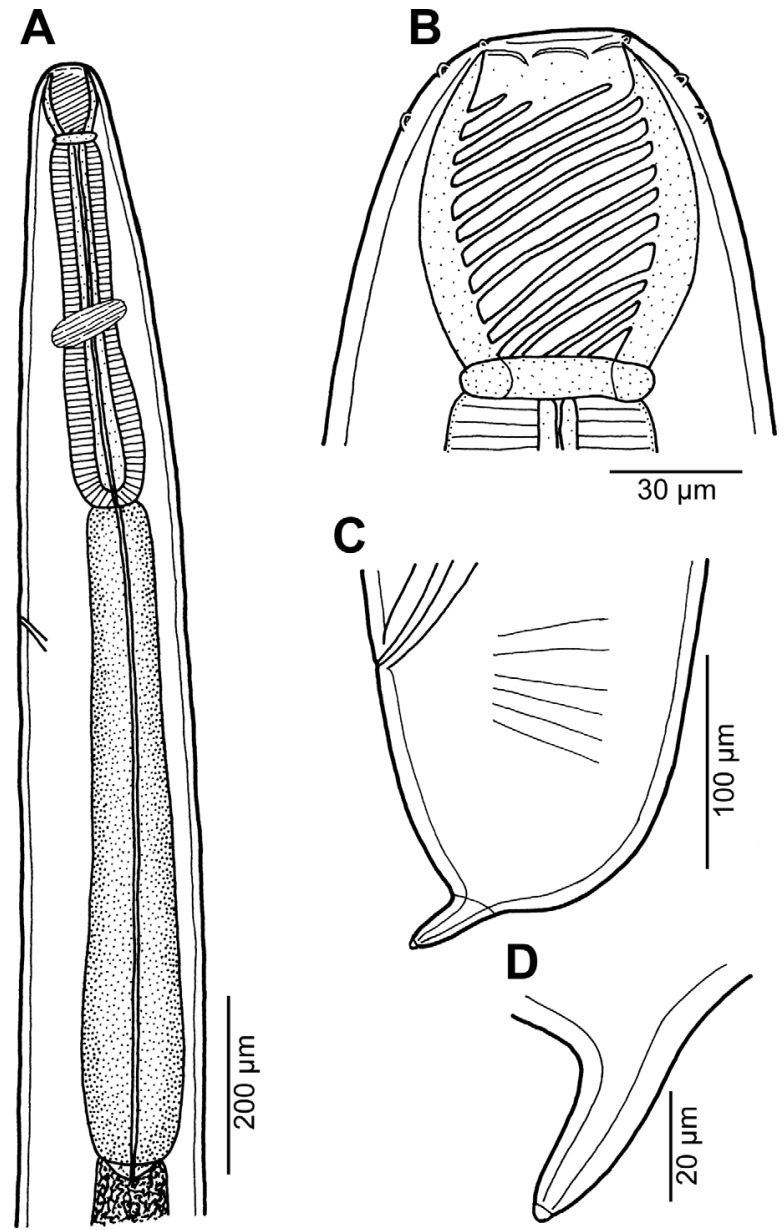

C

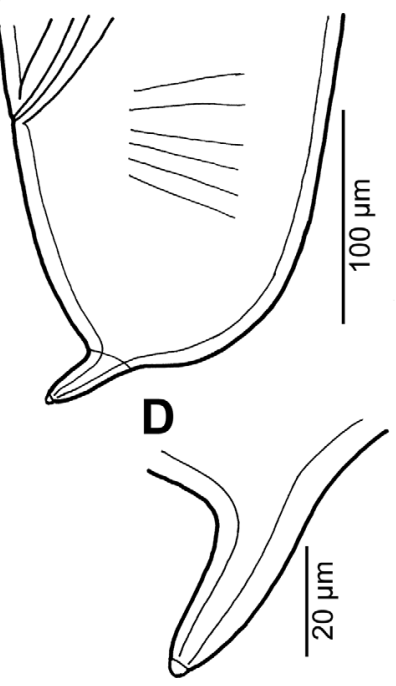

Figure 13. Procamallanus (Spirocamallanus) sp. 2 from Stegostoma fasciatum, subgravid female. (A) Anterior end, lateral view; (B) buccal capsule, lateral view; (C) tail, lateral view; (D) tail tip, lateral view.

Deposition of voucher specimen: Muséum National d'Histoire Naturelle, Paris (MNHN JNC 1529).

\section{Description}

Female (one ovigerous specimen): Length of body $16.17 \mathrm{~mm}$, maximum width 313 . Buccal capsule including basal ring 93 long, its width 69; basal ring 9 long and 48 wide. Maximum width/length ratio of buccal capsule $1: 1.35$. Spiral ridges 12, of which 3 incomplete (Fig. 13B). Length of muscular oesophagus 490, maximum width 105; length of glandular oesophagus 843, maximum width 138 (Fig. 13A); length ratio of muscular and glandular oesophagus 1:1.18. Length of entire oesophagus and buccal capsule representing $12 \%$ of body length. Nerve ring 313 from anterior extremity. Deirids not located. Excretory pore at short distance posterior to posterior end of muscular oesophagus, at 734 from anterior end of body (Fig. 13A). Vulva postequatorial, $8.34 \mathrm{~mm}$ from anterior extremity, at $52 \%$ of body length. Vulval lips not elevated. Vagina directed posteriorly from vulva. Uterus filled with small amount of eggs. Tail broad, rounded, its posterior end abruptly narrowed to form digital protrusion provided with 1 small

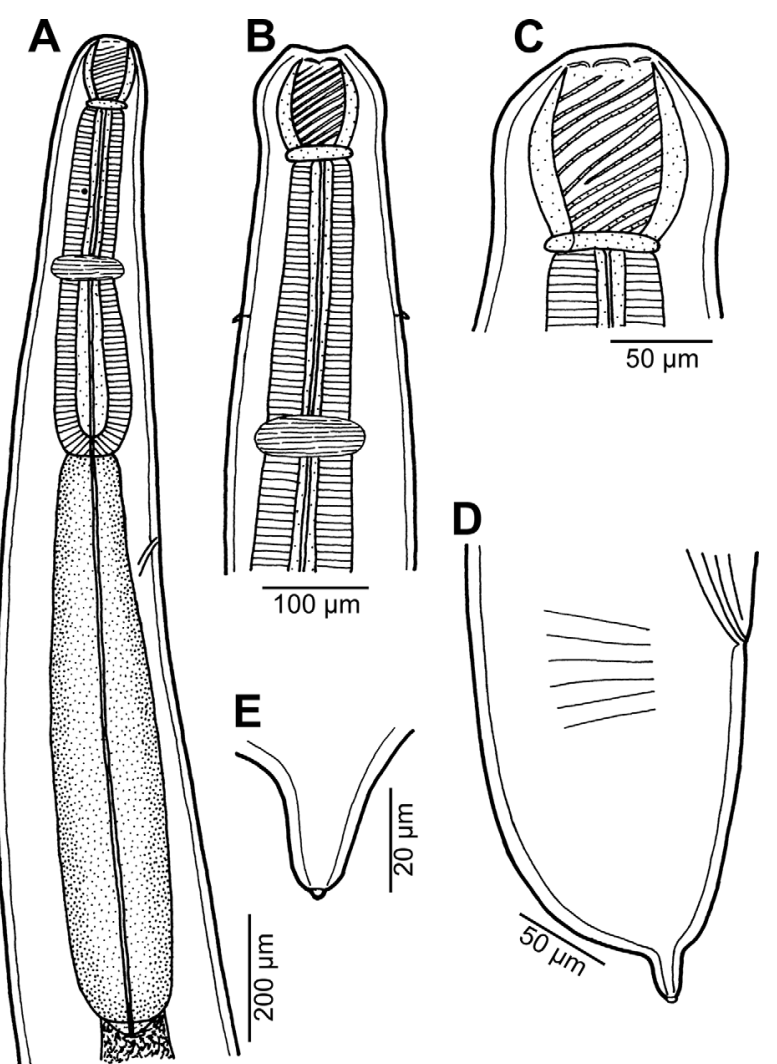

Figure 14. Procamallanus (Spirocamallanus) sp. 3 from Laticauda saintgironsi, subgravid female. (A, B) Anterior end, lateral and dorsoventral views, respectively; (C) buccal capsule, lateral view; (D) tail, lateral view; (E) tail tip, lateral view.

terminal cuticular knob; length of entire tail 163; digital protrusion 39 long, 15 wide (Figs. 13C and 13D).

\section{Remarks}

Due to availability of a single subgravid female, the species identification based on morphology is impossible. This species is characterized by the broadly rounded tail with a short projection without terminal cuticular spines. In this case, the shark apparently served as a postcyclic host, which had acquired the infection by feeding on the true definitive hosts (fish) of this nematode.

\section{Procamallanus (Spirocamallanus) sp. 3 Figure 14}

Host: New Caledonian sea krait Laticauda saintgironsi Cogger et Heatwole (Elapidae, Serpentes).

Site of infection: Collected from regurgitated digestive content of snake.

Locality: Île Amédée, off Nouméa, New Caledonia (collected 14 February 2011).

Prevalence and intensity: 1 snake infected/90 examined; 1 nematode.

Deposition of voucher specimen: Muséum National d'Histoire Naturelle, Paris, MNHN JNB011. 


\section{Description}

Female (one ovigerous specimen): Length of body 23.11 $\mathrm{mm}$, maximum width 462 . Buccal capsule including basal ring 114 long, its width 90; basal ring 12 long and 66 wide. Maximum width/length ratio of buccal capsule 1:1.27. Spiral ridges 13, of which 5 incomplete (Fig. 14C). Length of muscular oesophagus 598, maximum width 105; length of glandular oesophagus 1020, maximum width 192 (Fig. 14A); length ratio of muscular and glandular oesophagus 1:1.71. Length of entire oesophagus and buccal capsule representing $7 \%$ of body length. Nerve ring 367 from anterior extremity. Deirids small, situated 243 from anterior extremity, approximately at mid-way between base of buccal capsule and nerve ring (Fig. 14B). Excretory pore located short distance posterior to posterior end of muscular oesophagus, at 816 from anterior end of body (Fig. 14A). Vulva postequatorial, $11.90 \mathrm{~mm}$ from anterior extremity, at $52 \%$ of body length. Vulval lips not elevated. Vagina directed posteriorly from vulva. Uterus filled with many eggs. Tail broad, somewhat conical, its posterior end abruptly narrowed to form digit-like protrusion provided with one small terminal cuticular knob; length of entire tail 122; digit-like protrusion 27 long, 15 wide (Figs. 14D and 14E).

\section{Remarks}

The presence of a single subgravid female but no male makes species identification of this nematode impossible. As in the previous case, it is apparent that the actual definitive host is a fish and the sea-snake acts only as a postcyclic host, which acquired the infection by feeding on fish. The nematode was collected from the regurgitated digestive content of a snake induced by manipulation, no identifiable prey fish was recovered.

\section{Camallanus carangis Olsen, 1954}

Syns.: Camallanus marinus Schmidt et Kuntz, 1969; C. paracarangis Velasquez, 1980.

Hosts: Perciformes: Yellowtail scad Atule mate (Cuvier), yellowspotted trevally Carangoides fulvoguttatus (Forsskål), shadow trevally Carangoides dinema and bigeye scad Selar crumenophthalmus (Bloch) (all Carangidae), gold-saddle goatfish Parupeneus cyclostomus (Lacepède) and Indian goatfish Parupeneus indicus (Shaw) (both Mullidae), crimson jobfish Pristipomoides filamentosus (Valenciennes) (Lutjanidae), and tomato hind Cephalopholis sonnerati (Valenciennes), highfin grouper Epinephelus maculatus (Bloch), camouflage grouper Epinephelus polyphekadion (Bleeker) and red-tipped grouper Epinephelus retouti Bleeker (all Serranidae). Clupeiformes: dorab wolf-herring Chirocentrus dorab (Forsskål) (Chirocentridae). Gravid (larvigerous) females recorded only from $S$. crumenophthalmus. Only nematode fourth-stage larvae found in P. indicus and C. dorab.

In addition to fishes, a single fourth-stage larva of C. carangis was found in the regurgitated digestive content obtained from the New Caledonian sea krait Laticauda saintgironsi (Parasitological number JNB012) collected on Ilôt Signal, off Nouméa, on 28 February 2011.
Site of infection: Intestine.

Locality: Off Nouméa, New Caledonia.

Prevalence, intensity and details about fish: Atule mate: 1 fish infected/14 fish examined, 4 nematodes; infected fish, number JNC2963, 5 June 2009, Nouméa fishmarket, FL 318 mm, W 508 g. Carangoides fulvoguttatus: 1/15, 1 nematode; infected fish, number JNC3299, 28 January 2011, Nouméa fishmarket, FL 265 mm, W 409 g. Carangoides dinema: 1/9, 2 nematodes; infected fish, number JNC2882, 13 March 2009, Nouméa fishmarket, FL 293 mm, W 577 g. Selar crumenophthalmus: 2/10, 1-2 nematodes; infected fish, number JNC3043, 10 September 2009, Nouméa fishmarket, FL 227 mm, W $194 \mathrm{~g}$ and number JNC3125, Nouméa fishmarket, 25 November 2009, FL 240 mm, W 275 g. Parupeneus cyclostomus: 1/7, 2 nematodes; infected fish, number JNC1922, 22 August 2006, external reef near Ever Prosperity, off Nouméa, $22^{\circ} 27^{\prime} 28.8^{\prime \prime} \mathrm{S}, 166^{\circ} 21^{\prime} 50.4^{\prime \prime}$ E, FL $263 \mathrm{~mm}$, W $344 \mathrm{~g}$; fish deposited in the ichthyological collections as MNHN-IC-2006-1753. Parupeneus indicus: 1/11, 1 nematode; infected fish, number JNC1964, 21 September 2006, Nouméa fishmarket, FL 290 mm, W 560 g. Pristipomoides filamentosus: 1/7, 1 nematode; infected fish, number JNC2460, 26 February 2008, near Passe de Dumbéa, off Nouméa, $22^{\circ} 22^{\prime} \mathrm{S}, 166^{\circ} 14^{\prime} \mathrm{E}$, FL 360 mm, W 863 g. Cephalopholis sonnerati: 1/5, 3 nematodes; infected fish, number JNC2934, 12 May 2009, near Récif Toombo, off Nouméa, $22^{\circ} 31^{\prime} 40 \mathrm{~S}, 166^{\circ} 28^{\prime} 36 \mathrm{E}$, FL $474 \mathrm{~mm}$, W 1600 g. Epinephelus maculatus: 1/38, 1 nematode; infected fish, number JNC2157, 17 April 2007, External reef near Ever Prosperity, off Nouméa, $22^{\circ} 27^{\prime} 28.8^{\prime \prime}$ S, $166^{\circ} 21^{\prime} 50.4^{\prime \prime}$ E, FL $535 \mathrm{~mm}, \mathrm{~W} 2050$ g. Epinephelus polyphekadion: 1/3, 1 nematode; infected fish, number JNC3036, 8 September 2009, Passe de Dumbéa, off Nouméa, $22^{\circ} 21^{\prime} 232$ S, $166^{\circ}$ 14'236 E, FL 410 mm, W 1055 g. Epinephelus retouti: 1/2, 2 nematodes; infected fish, number JNC2181, 19 June 2009, near Récif Toombo, off Nouméa, FL 329 mm, W 575 g. Chirocentrus dorab: 1/11, 1 nematode; infected fish, number JNC3236, 9 September 2010, Nouméa fishmarket, FL $700 \mathrm{~mm}$, W $1538 \mathrm{~g}$.

Deposition of voucher specimens: Muséum National d'Histoire Naturelle, Paris (MNHN JNC364B, JNC1903, JNC1922, JNC1964, JNC2157C, JNC2181, JNC2882, JNC2934, JNC2960, JNC2963, JNC3036, JNC3236, JNB013).

\section{Remarks}

The general morphology of the present specimens corresponds to that of $C$. carangis, as redescribed by Moravec et al. [27], and, therefore, they are assigned to this species. Since $C$. carangis has been redescribed and illustrated in detail by Moravec et al. [27], who also described its characteristic fourth-stage larva, we refrain from repeating the description of this species, based on newly collected specimens. Nevertheless, their measurements are compared in Tables 1-3.

Camallanus carangis, originally described from Caranx $\mathrm{sp}$. in Fiji [36], is known as a parasite of carangids and fishes belonging to some other families in Hawaii, French Polynesia, the Philippines, in the Arabian, Arafura, South China and Red Seas and also from off New Caledonia [23]. The present findings of $C$. carangis in fishes Carangoides dinema, 
Table 1. Comparison of measurements of Camallanus carangis males from marine fishes in New Caledonia.

\begin{tabular}{lcccc}
\hline Host & Atule mate & Carangoides fulvoguttatus & Cephalopholis sonnerati & Epinephelus retouti \\
\hline No. of specimens & 2 & 1 & 3 & 1 \\
Body length (in mm) & $12.17-13.67$ & 6.90 & $9.93-11.32$ & frag. 5.40 \\
Body width & $299-354$ & 218 & $258-326$ & 258 \\
Buccal capsule - length & $165-189$ & 162 & $183-186$ & 159 \\
Buccal capsule - width & $159-189$ & 156 & $156-183$ & 165 \\
No. of ridges & $33-35$ & 38 & $34-35$ & 40 \\
Basal ring - length & $21-24$ & 27 & $27-33$ & 27 \\
Basal ring - width & $90-105$ & 90 & $96-102$ & 93 \\
Length of tridents & 150 & 150 & $171-219$ & 153 \\
Oesoph. cup - length & $21-30$ & 30 & $30-36$ & 36 \\
Oesoph. cup - width & $24-33$ & 39 & $36-39$ & 30 \\
Musc. oesoph. - length & $1183-1414$ & 1020 & $122-136$ & 1102 \\
Musc. oesoph. - width & $122-136$ & 81 & $1034-1238$ & 84 \\
Gland. oesoph. - length & $1156-1333$ & 857 & $122-136$ & 1673 \\
Gland. oesoph. - width & 136 & 96 & $1: 0.88-0.93$ & 90 \\
Musc./gland. oesoph. length ratio & $1: 0.94-0.98$ & $1: 084$ & $23-24$ & $1: 0.79$ \\
$\%$ of buc. c. and oesoph. of body & 21 & 30 & $1115-1251$ & - \\
Excretory pore & $1047-1387$ & $?$ & $294-312$ & - \\
Right spicule & $306-309$ & 300 & $84-117$ & - \\
Tail & $75-81$ & 102 & & \\
\hline
\end{tabular}

Table 2. Comparison of measurements of Camallanus carangis females from marine fishes in New Caledonia.

\begin{tabular}{|c|c|c|c|c|c|c|}
\hline Host & $\begin{array}{l}\text { Atule } \\
\text { mate }\end{array}$ & $\begin{array}{c}\text { Carangoides } \\
\text { dinema }\end{array}$ & $\begin{array}{c}\text { Selar } \\
\text { crumenophthalmus }\end{array}$ & $\begin{array}{l}\text { Parupeneus } \\
\text { cyclostomus }\end{array}$ & $\begin{array}{l}\text { Pristipomoides } \\
\text { filamentosus }\end{array}$ & $\begin{array}{l}\text { Epinephelus } \\
\text { retouti }\end{array}$ \\
\hline No. of specimens & 1 (grav.) & 2 (subgr.) & 3 (gr., subgr.) & 2 (subgr.) & 1 (subgr.) & 1 (subgr.) \\
\hline Body length (in mm) & 14.93 & $4.75-7.00$ & $14.63-17.00$ & $10.23-11.13$ & 14.89 & 10.95 \\
\hline Body width & 340 & $163-245$ & $381-517$ & $340-408$ & 313 & 354 \\
\hline Buccal capsule - length & 195 & $159-180$ & $186-225$ & 159 & 150 & 183 \\
\hline Buccal capsule - width & 198 & $144-180$ & $204-233$ & 144 & 159 & 195 \\
\hline No. of ridges & 32 & $37-40$ & $35-50$ & $?$ & 32 & 44 \\
\hline Basal ring - length & 30 & 27 & $24-36$ & $21-24$ & 24 & 30 \\
\hline Basal ring - width & 111 & 96 & $108-117$ & $96-99$ & 90 & 108 \\
\hline Length of tridents & 141 & 135 & $195-240$ & $105-195$ & 141 & 153 \\
\hline Oesoph. cup - length & 27 & $24-30$ & $30-39$ & 33 & 15 & 36 \\
\hline Oesoph. cup - width & 36 & $30-36$ & $39-45$ & 36 & 33 & 30 \\
\hline Musc. oesoph. - length & 1550 & $721-1047$ & $1333-1850$ & $816-979$ & 911 & 1469 \\
\hline Musc. oesoph. - width & 136 & 90 & $123-177$ & $122-136$ & 163 & 122 \\
\hline Gland. oesoph. - length & 1333 & $653-911$ & $1156-1659$ & $775-925$ & 993 & 1156 \\
\hline Gland. oesoph. - width & 136 & $96-99$ & $114-190$ & $122-163$ & 163 & 136 \\
\hline $\begin{array}{l}\text { Musc./gland. oesoph. } \\
\text { length ratio }\end{array}$ & $1: 0.86$ & $1: 0.87-0.91$ & $1: 0.87-0.98$ & 1:0.94-0.95 & $1: 1.09$ & $1: 0.79$ \\
\hline $\begin{array}{l}\% \text { of buc. c. and oesoph. } \\
\text { of body }\end{array}$ & 21 & $22-40$ & $18-25$ & $17-19$ & 14 & 26 \\
\hline Excretory pore & 1401 & $707-911$ & 1591 & $?$ & $?$ & 1224 \\
\hline $\begin{array}{l}\text { Vulva from ant. end (in } \\
\mathrm{mm} \text { ) }\end{array}$ & 7.51 & $2.56-3.93$ & $7.13-8.80$ & 4.92 & 7.00 & 5.74 \\
\hline$\%$ of vulva of body & 50 & $54-56$ & $49-55$ & 48 & 47 & 52 \\
\hline Tail & 272 & $90-136$ & 225 & $144-147$ & 177 & 190 \\
\hline
\end{tabular}

C. fulvoguttatus, Cephalopholis sonnerati, Chirocentrus dorab, Epinephelus maculatus, E. retouti, Parupeneus cyclostomus, $P$. indicus, Pristipomoides filamentosus and Selar crumenophthalmus, as well as in the sea-snake Laticauda saintgironsi, represent new host records.

From New Caledonian waters, C. carangis was previously reported from marine perciform fishes Carangoides chrysophrys (Cuvier) and C. hedlandensis (Whitley) (Carangidae),
Nemipterus furcosus (Valenciennes) (Nemipteridae), and Parupeneus ciliatus (Lacepède) and Upeneus vittatus (Forsskål) (both Mullidae) [23, 27]. The present survey extends considerably the range of hosts of $C$. carangis in New Caledonia, now including 15 fish species of the perciform families Carangidae, Lutjanidae, Mullidae, Nemipteridae and Serranidae, and a representative of the clupeiform family Chirocentridae. Of them, however, gravid (= larvigerous) females of this nematode have 
Table 3. Comparison of measurements of Camallanus carangis four-stage larvae from fish and reptilian hosts in New Caledonia.

\begin{tabular}{lccc}
\hline Host & $\begin{array}{c}\text { Parupeneus } \\
\text { indicus }\end{array}$ & $\begin{array}{c}\text { Chirocentrus } \\
\text { dorab }\end{array}$ & $\begin{array}{c}\text { Laticauda } \\
\text { saintgironsi }\end{array}$ \\
\hline No. of specimens & 1 & 1 & 1 \\
Body length (in mm) & 3.13 & 4.12 & 1.31 \\
Body width & 136 & 109 & 122 \\
Buccal capsule - length & 84 & 105 & 102 \\
Buccal capsule - width & 69 & 69 & 90 \\
No. of ridges & 17 & $?$ & 18 \\
Basal ring - length & 12 & 21 & 24 \\
Basal ring - width & 51 & 51 & 54 \\
Length of prongs & 78 & 123 & 117 \\
Oesoph. cup - length & 18 & 24 & 18 \\
Oesoph. cup - width & 21 & 21 & 21 \\
Musc. oesoph. - length & 435 & 571 & 517 \\
Musc. oesoph. - width & 63 & 60 & 45 \\
Gland. oesoph. - length & 394 & 490 & 435 \\
Gland. oesoph. - width & 72 & 60 & 45 \\
Musc./gland. oesoph. & $1: 091$ & $1: 0.86$ & $1: 0.84$ \\
$\quad$ length ratio & & & \\
\% of buc. c. and & 2 & 28 & 81 \\
$\quad$ oesoph. of body & & & \\
Tail & 72 & 147 & 54 \\
\hline
\end{tabular}

* Sea-snake (Reptilia).

so far been recorded only from the carangid S. crumenophthalmus and the mullid $U$. vittatus, confirming thus that these fishes serve as the true definitive hosts for this parasite. Apparently, some of the hosts recorded serve only as paratenic, paradefinitive or postcyclic hosts [34], as is known, for example, for Camallanus lacustris (Zoega, 1776) or C. oxycephalus Ward et Magath, 1916, parasites of freshwater fishes in the Holarctic [20, 21, 44]. The present record of the $C$. carangis fourth-stage larva in the digestive tract of a sea-snake indicates that the snake acquired this infection while feeding on fish.

To date, $C$. carangis is the only representative of Camallanus parasitizing marine fishes in New Caledonian waters. Another congeneric species, $C$. cotti, a parasite of freshwater fishes, was introduced into New Caledonia [24].

\section{Discussion}

All species of Procamallanus (Spirocamallanus) reported in this study belong to the morphological group of nematodes characterized by the presence of wide caudal alae, three pairs of pedunculate preanal papillae and two unequal spicules; as mentioned above, this mostly includes parasites of marine perciform fishes [37, 38]. Many species with these characteristics have been, often inadequately, described from different geographical zones, which makes a thorough comparison of them almost impossible. This situation is more complicated by the fact that some taxonomically important morphological features of these nematodes (e.g., the shape and position of deirids, excretory pore or the number and distribution of postanal papillae) are not easily observed under the light microscope and, consequently, that some insufficiently described species are reported from numerous, often unrelated hosts.
According to Petter et al. [38], Rigby and Adamson [39] and Moravec et al. [29], the shape and structure of the female tail of these nematodes appear to be constant within a species in Procamallanus (Spirocamallanus). Most species possess 2-4 terminal spines on the digital caudal projection in the female, whereas these are lacking only in a few species. Nevertheless, the morphology of all these species is rather similar. Although the division of Procamallanus (Spirocamallanus) species according to geographical zones by Andrade-Salas et al. [2] has been used by some authors [22, 27, 31, 39, 40] for the comparison of species, recent detailed morphological studies of some of these nematodes, including SEM, indicate a certain degree of their host specificity (approximately at the level of fish family), which should also be considered when evaluating these nematodes. This is supported by the present findings.

A quite different situation is regarding the species C. carangis, which, in New Caledonia, has been reported from 15 host species belonging to six fish families. This is not surprising, because a low degree of host specificity is well known for some other species of Camallanus, for example C. cotti, C. lacustris, C. oxycephalus or C. truncatus (Rudolphi, 1814). This is related with the circulation of these parasites in the environment, when different categories of hosts are employed during the development of these nematodes, i.e., different fishes may play a role of paratenic, definitive, paradefinitive or postcyclic hosts. Some aquatic snakes were found to be postcyclic hosts of the European species C. lacustris and C. truncatus [21].

Our records might be the first parasitological records for L. saintgironsi, a species recently described [6]; the species is endemic to New Caledonia [8] and sympatric with another species, L. laticaudata (Linnaeus) [5]. Its diet consists of non-spiny anguilliform fish, with the lipspot moray Gymnothorax chilospilus Bleeker representing about half of the prey [5]. The present records of Procamallanus (S.) sp. 3 subgravid female and that of $C$. carangis four-stage larva in the sea snakes L. saintgironsi indicate that these hosts acquired the infection with camallanids by feeding on fish hosts - probably morays - of these nematodes. Camallanids may survive in the digestive tract of fish-eating snakes for a long period (up to several months), as observed in $C$. truncatus overwintering in the European colubrid snake Natrix tessellata (Laurenti) [19]; in this case, it served as the postcyclic host. Regarding the above-mentioned camallanids in L. saintgironsi, these snakes served as postcyclic and paratenic hosts, respectively.

Acknowledgements. We wish to thank all colleagues and students who participated in the parasitological survey over the years; detailed lists were provided in previous papers [12-14]. Fish were identified, generally from photographs, by experienced ichthyologists, including Kunio Amaoka, Ronald Fricke, Samuel Iglesias, John E. Randall, and Bernard Séret. Marine Briand collected the snakes and performed their parasitological survey. Thanks are also due to the Laboratory of Electron Microscopy, Institute of Parasitology, Biology Centre CAS, institution supported by the MEYS CR (LM2015062 Czech-BioImaging) for their support with obtaining scientific data presented in this paper, and to Blanka Škoríková of the same Institute for help with the illustrations. This study was partly supported by the institutional support of the Institute of Parasitology, BC AS CR (585110/9500). 


\section{Conflict of interest}

The Editor-in-Chief of Parasite is one of the authors of this manuscript. COPE (Committee on Publication Ethics, http:// publicationethics.org), to which Parasite adheres, advises special treatment in these cases. In this case, the peer-review process was handled by an Invited Editor, Jérôme Depaquit.

\section{References}

1. Anderson RC, Chabaud AG, Willmott S, Editors. 2009. Keys to the nematode parasites of vertebrates. Archival volume. Wallingford: CAB International. p. 463.

2. Andrade-Salas O, Pineda-López RF, García-Magaña L. 1994. Spirocamallanus rebecae sp. n. (Nematoda: Camallanidae) from freshwater fishes in south-eastern Mexico. Folia Parasitologica, 41, 259-270.

3. Annereaux RF. 1946. A new nematode, Procamallanus pereirai, with a key to the genus. Transactions of the American Microscopical Society, 65, 299-303.

4. Briand MJ, Letourneur Y, Bonnet X, Wafo E, Fauvel T, Brischoux F, Guillou G, Bustamante P. 2014. Spatial variability of metallic and organic contamination of anguilliform fish in New Caledonia. Environmental Science and Pollution Research, 21, 4576-4591.

5. Brischoux F, Bonnet X, Shine R. 2009. Determinants of dietary specialization: a comparison of two sympatric species of sea snakes. Oikos, 118, 145-151.

6. Cogger HG, Heatwole HG. 2006. Laticauda frontalis (de Vis, 1905) and Laticauda saintgironsi $n$. sp. from Vanuatu and New Caledonia (Serpentes: Elapidae: Laticaudinae) - a new lineage of sea kraits? Records of the Australian Museum, 58, 245-256.

7. Froese R, Pauly D, Editors. 2019. FishBase. World Wide Web Electronic Publication. http://www.fishbase.org, 09/2019.

8. Gherghel I, Papes M, Brischoux F, Sahlean T, Strugariu A. 2016. A revision of the distribution of sea kraits (Reptilia, Laticauda) with an updated occurrence dataset for ecological and conservation research. ZooKeys, 569, 135-148.

9. González-Solís D, Moravec F, Vidal-Martínez VM. 2002. Procamallanus (Spirocamallanus) chetumalensis n. sp. (Nematoda: Camallanidae) from the Mayan sea catfish, Ariopsis assimilis, off the Caribbean coast of Mexico. Journal of Parasitology, 88, 765-768.

10. Ivashkin VM, Sobolev AA, Khromova LA. 1971. Camallanata of animals and man and the diseases caused by them. Osnovy Nematodologii 22. Moscow: Nauka. p. 388. [In Russian].

11. Johnston TH, Mawson PM. 1940. Some nematodes parasitic in Australian freshwater fish. Transactions of the Royal Society of South Australia, 64, 340-352.

12. Justine J-L, Beveridge I, Boxshall GA, Bray RA, Miller TL, Moravec F, Trilles J-P, Whittington ID. 2012. An annotated list of fish parasites (Isopoda, Copepoda, Monogenea, Digenea, Cestoda, Nematoda) collected from snappers and bream (Lutjanidae, Nemipteridae, Caesionidae) in New Caledonia confirms high parasite biodiversity on coral reef fish. Aquatic Biosystems, 8, 22.

13. Justine J-L, Beveridge I, Boxshall GA, Bray RA, Moravec F, Trilles J-P, Whittington ID. 2010. An annotated list of parasites (Isopoda, Copepoda, Monogenea, Digenea, Cestoda and Nematoda) collected in groupers (Serranidae, Epinephelinae) in New Caledonia emphasizes parasite biodiversity in coral reef fish. Folia Parasitologica, 57, 237-262.
14. Justine J-L, Beveridge I, Boxshall GA, Bray RA, Moravec F, Whittington ID. 2010. An annotated list of fish parasites (Copepoda, Monogenea, Digenea, Cestoda and Nematoda) collected from emperors and emperor bream (Lethrinidae) in New Caledonia further highlights parasite biodiversity estimates on coral reef fish. Zootaxa, 2691, 1-40.

15. Justine J-L, Briand MJ, Bray RA. 2012. A quick and simple method, usable in the field, for collecting parasites in suitable condition for both morphological and molecular studies. Parasitology Research, 111, 341-351.

16. Luque JL, Aguiar JC, Vieira FM, Gibson DI, Santos CP. 2011. Checklist of Nematoda associated with the fishes of Brazil. Zootaxa, 3082, 1-88.

17. Machida M, Taki Y. 1985. A new species of Spirocamallanus (Nematoda, Camallanidae) from rabbitfish in the Philippines. Bulletin of the National Science Museum, Series A, 11, 53-56.

18. Mašová Š, Baruš V, Moravec F. 2011. New morphological data on the first-stage larvae of two Procamallanus species (Nematoda: Camallanidae) based on SEM studies. Folia Parasitologica, 58, 318-321.

19. Moravec F. 1963. A contribution to the knowledge of the helminth fauna of our reptiles. Publications de la Faculté des Sciences de l'Université J. E. Purkyně Brno, 446, 353-396. [In Czech with Russian and English summaries].

20. Moravec F. 1971. On the problem of host specificity, reservoir parasitism and secondary invasions of Camallanus lacustris (Nematoda: Camallanidae). Helminthologia, 10, 107-114.

21. Moravec F. 2013. Parasitic nematodes of freshwater fishes of Europe. Prague: Academia. p. 601. Revised second edition.

22. Moravec F, Cruz-Lacierda ER, Nagasawa K. 2004. Two Procamallanus spp. (Nematoda, Camallanidae) from fishes in the Philippines. Acta Parasitologica, 49, 309-318.

23. Moravec F, Gey D, Justine J-L. 2016. Nematode parasites of four species of Carangoides (Osteichthyes: Carangidae) in New Caledonian waters, with a description of Philometra dispar $\mathrm{n}$. sp. (Philometridae). Parasite, 23, 40.

24. Moravec F, Justine J-L. 2006. Camallanus cotti (Nematoda: Camallanidae), an introduced parasite of fishes in New Caledonia. Folia Parasitologica, 53, 287-296.

25. Moravec F, Justine J-L. 2011. New data on the morphology of Procamallanus (Procamallanus) annulatus and Procamallanus (Spirocamallanus) monotaxis (Nematoda: Camallanidae) from marine fishes off New Caledonia. Helminthologia, 48, 41-50.

26. Moravec F, Justine J-L. 2017. Two new species of nematode parasites, Cucullanus epinepheli sp. n. (Cucullanidae) and Procamallanus (Spirocamallanus) sinespinis sp. n. (Camallanidae), from marine serranid and haemulid fishes off New Caledonia. Folia Parasitologica, 64, 011.

27. Moravec F, Justine J-L, Rigby MC. 2006. Some camallanid nematodes from marine perciform fishes off New Caledonia. Folia Parasitologica, 53, 223-239.

28. Moravec F, Justine J-L, Würtz J, Taraschewski H, Sasal P. 2006. A new species of Procamallanus (Nematoda: Camallanidae) from Pacific eels (Anguilla spp.). Journal of Parasitology, 92, 130-137.

29. Moravec F, Salgado-Maldonado G, Caspeta-Mandujano J. 2000. Three new Procamallanus (Spirocamallanus) species from freshwater fishes in Mexico. Journal of Parasitology, 86, 119-127.

30. Moravec F, Sheeba S, Kumar AB. 2013. Observations on nematodes from the Indonesian shortfin eel Anguilla bicolor bicolor McClelland in India, including a revalidation of Heliconema ahiri Karve, 1941 (Physalopteridae). Acta Parasitologica, 58, 496-503. 
31. Moravec F, Taraschewski H, Thairungroj Anantaphruti M, Maipanich W, Laoprasert T. 2006. Procamallanus (Spirocamallanus) anguillae sp. n. (Camallanidae) and some other nematodes from the Indonesian shortfin eel Anguilla bicolor in Thailand. Parasitology Research, 100, 69-75.

32. Moravec F, Thatcher VE. 1997. Procamallanus (Denticamallanus subgen. n.) dentatus n. sp. (Nematoda: Camallanidae) from the characid fish, Bryconops alburnoides, in the Brazilian Amazon. Parasite, 4, 239-243.

33. Noble ER. 1966. A new camallanid nematode from Hawaii. Pacific Science, 20, 360-366.

34. Odening K. 1976. Conception and terminology of hosts in parasitology. Advances in Parasitology, 14, 1-93.

35. Olsen LS. 1952. Some nematodes parasitic in marine fishes. Publications of the Institute of Marine Science, University of Texas, 2, 173-215.

36. Olsen LS. 1954. A new species of Camallanus (Nematoda) from a Fijian marine fish. Transactions of the American Microscopical Society, 73, 258-260.

37. Petter A-J. 1979. Essai de classification de la sous-famille des Procamallaninae (Nematoda, Camallanidae). Bulletin du Muséum National d'Histoire Naturelle, Paris, Section A, Zoologie, 1, 991-1008.

38. Petter A-J, Golvan YJ, Tcheprakoff R. 1977. Nématodes de Poissons de rivière en Guadeloupe. Bulletin du Muséum National d'Histoire Naturelle, Paris, $3^{\mathrm{e}}$ série, No. 428, Section A, Zoologie, 298, 159-171.

39. Rigby MC, Adamson ML. 1997. Spirocamallanus species of French Polynesian coral reef fishes. Canadian Journal of Zoology, 75, 1270-1279.
40. Rigby MC, Font WF. 1997. Redescription and range extension of Spirocamallanus istiblenni Noble, 1966 (Nematoda: Camallanidae) from coral reef fishes in the Pacific. Journal of the Helminthological Society of Washington, 64, 227-233.

41. Rigby MC, Rigby E. 2014. Order Camallanida: Superfamilies Anguillicoloidea and Camallanoidea, in Handbook of Zoology - Gastrotricha, Cycloneuralia and Gnathifera. Volume 2: Nematoda, Schmidt-Rhaesa A, Editor. Walter De Gruyter GmbH: Berlin/Boston. p. 637-659.

42. Sardella CJ, Pereira FB, Luque JL. 2017. Redescription and first genetic characterization of Procamallanus (Spirocamallanus) macaensis Vicente \& Santos, 1972 (Nematoda: Camallanidae), including re-evaluation of the species of Procamallanus (Spirocamallanus) from marine fishes off Brazil. Systematic Parasitology, 94, 657-668.

43. Shamsi S, Briand MJ, Justine J-L. 2017. Occurrence of Anisakis (Nematoda: Anisakidae) larvae in unusual hosts in Southern hemisphere. Parasitology International, 66, 837-840.

44. Stromberg PC, Crites JL. 1974. The life cycle and development of Camallanus oxycephalus Ward et Magath, 1916 (Nematoda: Camallanidae). Journal of Parasitology, 60, 117-124.

45. Vicente JJ, Santos E. 1972. Sobre um novo nematódeo camalanídeo parasito de peixe marinho (Nematoda, Camallanoidea). Atas da Sociedade de Biologia do Rio de Janeiro, 15, $145-147$.

46. Yooyen T, Moravec F, Wongsawad C. 2011. Two new sibling species of Procamallanus (Spirocamallanus) (Nematoda: Camallanidae) from marine fishes in the Gulf of Thailand. Journal of Parasitology, 97, 931-938.

Cite this article as: Moravec F \& Justine J-L. 2019. New species and new records of camallanid nematodes (Nematoda, Camallanidae) from marine fishes and sea snakes in New Caledonia. Parasite 26, 66.

- PARASTE

An international open-access, peer-reviewed, online journal publishing high quality papers on all aspects of human and animal parasitology

Reviews, articles and short notes may be submitted. Fields include, but are not limited to: general, medical and veterinary parasitology; morphology, including ultrastructure; parasite systematics, including entomology, acarology, helminthology and protistology, and molecular analyses; molecular biology and biochemistry; immunology of parasitic diseases; host-parasite relationships; ecology and life history of parasites; epidemiology; therapeutics; new diagnostic tools.

All papers in Parasite are published in English. Manuscripts should have a broad interest and must not have been published or submitted elsewhere. No limit is imposed on the length of manuscripts.

Parasite (open-access) continues Parasite (print and online editions, 1994-2012) and Annales de Parasitologie Humaine et Comparée (1923-1993) and is the official journal of the Société Française de Parasitologie. 